\title{
What is the effect of diet and/or exercise interventions on behavioural compensation in non-exercise physical activity and related energy expenditure of free-living adults? A systematic review
}

\author{
Analiza M. Silva ${ }^{1 *}$, Pedro B. Júdice ${ }^{1}$, Eliana V. Carraça ${ }^{1}$, Neil King ${ }^{2}$, Pedro J. Teixeira ${ }^{1}$ and \\ Luís B. Sardinha ${ }^{1}$ \\ ${ }^{1}$ Exercise and Health Laboratory, Interdisciplinary Centre for the Study of Human Performance (CIPER), Faculdade \\ Motricidade Humana, Universidade Lisboa, 1499-002 Cruz Quebrada, Portugal \\ ${ }^{2}$ Institute of Health and Biomedical Innovation, Queensland University of Technology, Brisbane, QLD 4059, Australia \\ (Submitted 24 November 2017 - Final revision received 24 February 2018 - Accepted 16 March 2018)
}

\section{Abstract}

Non-exercise physical activity (NEPA) and/or non-exercise activity thermogenesis (NEAT) reductions may occur from diet and/or exerciseinduced negative energy balance interventions, resulting in less-than-expected weight loss. This systematic review describes the effects of prescribed diet and/or physical activity (PA)/exercise on NEPA and/or NEAT in adults. Studies were identified from PubMed, webof-knowledge, Embase, SPORTDiscus, ERIC and PsycINFO searches up to 1 March 2017. Eligibility criteria included randomised controlled trials (RCT), randomised trials (RT) and non-randomised trials (NRT); objective measures of PA and energy expenditure; data on NEPA, NEAT and spontaneous PA; $\geq 10$ healthy male/female aged $>18$ years; and $\geq 7 \mathrm{~d}$ length. The trial is registered at PROSPERO-2017-CRD42017052635. In all, thirty-six articles (RCT-10, RT-9, NRT-17) with a total of seventy intervention arms (diet, exercise, combined diet/exercise), with a total of 1561 participants, were included. Compensation was observed in twenty-six out of seventy intervention arms (fifteen studies out of thirty-six reporting declines in NEAT (eight), NEPA (four) or both (three)) representing 63, 27 and 23\% of diet-only, combined diet/exercise, and exercise-only intervention arms, respectively. Weight loss observed in participants who decreased NEAT was double the weight loss found in those who did not compensate, suggesting that the energy imbalance degree may lead to energy conservation. Although these findings do not support the hypothesis that prescribed diet and/or exercise results in decreased NEAT and NEPA in healthy adults, the underpowered trial design and the lack of state-of-the-art methods may limit these conclusions. Future studies should explore the impact of weight-loss magnitude, energetic restriction degree, exercise dose and participant characteristics on NEAT and/or NEPA.

Key words: Energy balance: Physical activity: Energy expenditure: Behavioural compensation: Weight loss: Free-living physical activity

Weight loss in the absence of disease or surgical intervention can only occur as the result of a chronic negative imbalance between energy intake (EI) and energy expenditure (EE). Although apparently simple, energy balance regulation is a dynamic process that requires a better understanding for evidence-based and realist interventions ${ }^{(1)}$.

Metabolic and behavioural compensations have been observed in response to diet and/or exercise interventions designed to induce changes in the energy balance components ${ }^{(2-4)}$. It is recognised that weight loss induced from a negative energy balance reduces over time, as energetic demands are attenuated, mitigating an indefinite exposure to energy balance deficit ${ }^{(5)}$. Although metabolic adaption to weight loss occurs ${ }^{(5,6)}$, behavioural compensations, that is compensation for an energy balance intervention through behaviour changes, may also occur. Indeed, behavioural compensation resulting from creating a negative energy balance includes a reduction in voluntary EE and/or an increase in EI in the absence of a strict control ${ }^{(7)}$. To better clarify voluntary EE, important concepts should be addressed, such as physical activity EE (PAEE) that represents the overall energy expended to move the body, further divided into structured physical

Abbreviations: ACC, accelerometer; DLW, doubly labelled water; EE, energy expenditure; EI, energy intake; ExEE, exercise energy expenditure; HR, heart rate; NEAT, non-exercise activity thermogenesis; NEPA, non-exercise physical activity; NRT, non-randomised trial; PA, physical activity; PAEE, physical activity energy expenditure; RCT, randomised controlled trial; REE, resting energy expenditure; RT, randomised trials; SMR, sleeping metabolic rate; TEE, total energy expenditure; TEF, thermic effect of food.

* Corresponding author: A. M. Silva, email analiza@fmh.ulisboa.pt 
activity (PA) (exercise) or non-exercise activity thermogenesis (NEAT). Non-exercise PA (NEPA) refers to the physical motion of the body in activities that do not pertain to volitional exercise, including all activities of daily living (fidgeting, maintaining posture and ambulation), whereas NEAT defines the EE associated with these activities ${ }^{(8)}$. However, the role of NEPA and/or NEAT on compensation from exercise and/or diet-induced weight loss is less well understood.

A recent systematic review with meta-analysis indicated no mean changes in NEPA during exercise training ${ }^{(9)}$. However, the authors reported that session duration, intervention length, age and sex influenced changes in NEPA during exercise training ${ }^{(9)}$. Washburn et al. ${ }^{(10)}$ indicated that more data from adequately powered trials using objective measurements are required to improve the understanding of the effects of exercise-induced weight loss on NEAT and NEPA. Measurement of EI and EE, including all components of EE, objective measurement of PA and accurate measurement of changes in body energy stores, must be included in such studies.

Relatively few studies have investigated the effect of energy restriction on free-living NEPA and NEAT, possible owing to the cost and burden of measuring PA accurately in participants' habitual environment. Furthermore, many of the findings are contradictory. No changes in posture allocation (time spent reclining or sitting $v$. standing or ambulating) were observed when obese people lost weight ${ }^{(11)}$, whereas other studies only found trends towards decreases in PA among non-obese weightreduced men ${ }^{(12,13)}$. In contrast, other groups found a decrease in PA and corresponding EE during an energy restriction diet, with inclusion of exercise training ${ }^{(14,15)}$. Three randomised controlled trials (RCT) under the Comprehensive Assessment of Long-Term Effects of Reducing Intake of Energy (CALERIE) study found that energy restriction significantly decreased NEAT, but not NEPA ${ }^{(16)}$.

Dhurandhar et al. ${ }^{(17)}$ provided a systematic review with metaanalysis using a mathematical modelling approach concluding that there is substantial compensation in both dietary and exercise interventions designed to induce weight loss. The authors identified a possible range of behavioural and metabolic compensations that can be very difficult to quantify, but which may reduce the expected amount of weight loss after a given intervention ${ }^{(17)}$. The extent to which this compensation is due to changes in NEPA or NEAT is unclear. There is insufficient evidence to definitively answer the question of whether diet or exercise-induce weight loss leads to compensatory reductions in NEAT and NEPA, as a result of increases in sedentary behaviour, decreases in overall PA or both. So far no systematic review has covered both exercise and diet, and their combined and independent effects on compensatory activity. The aim of this systematic review is to describe the effects of diet and/or exercise energy balance interventions on behavioural compensation in NEPA and/or related decreases in NEAT of free-living adults.

\section{Methods}

\section{Criteria for study eligibility: studies and participants}

In this review, articles reporting changes in compensatory behaviours occurring during or as a result of diet and/or exercise interventions, designed to intervene in one or more components of the energy balance equation, were retrieved. To be included, studies had to fulfil all of the following criteria: (1) adult samples (>18 years), regardless of sex; (2) $n>10$ participants; (3) an intervention period of at least 1 week; (4) be published in English language; (5) include objective measures of total EE (TEE) and/or PA (doubly labelled water (DLW), indirect calorimetry, accelerometer (ACC), pedometer, inclinometer); and (6) be a clinical trial. In turn, studies involving participants taking medication or having diseases/conditions known to affect metabolism/ weight (cancer, thyroid disease, diabetes, bariatric surgery, pregnancy, total parenteral nutrition, HIV/AIDS, organ transplant, Prader-Willi Syndrome, polycystic ovary syndrome, chronic obstructive pulmonary disease or acute illnesses, such as infections or traumatic injury) were excluded. The current review is registered on PROSPERO (PROSPERO 2017 CRD42017052635).

\section{Information sources and search strategy}

A comprehensive search of peer-reviewed articles published until 1 March 2017 (including online ahead of print publications) was conducted in the following electronic databases: Pubmed, PsycINFO, Embase, CINAHL, Cochrane Library, ERIC and SPORTDiscus. Searches included all meaningful combinations of the following sets of terms: (i) terms concerning the population of interest (e.g. adults, obese, overweight); (ii) terms concerning the intervention(s) of interest (e.g. diet or energetic restriction, PA or exercise, weight or body fat loss/change, behaviour change or lifestyle intervention); (iii) terms representing the outcomes of interest (e.g. NEPA, spontaneous PA, NEAT, compensatory response/behaviour); and (iv) terms concerning the study design (e.g. trial, experimental, treatment). A complete list of search strategies can be obtained from the authors, whereas a search strategy example for Pubmed is provided as an additional file (online Supplementary material SI). Other sources included manual cross-referencing of literature cited in prior reviews and retrieved studies, and hand-searches of the content of key scientific journals.

\section{Study selection and data processing}

All abstracts identified from the literature searches were screened for potential inclusion eligibility by one author (P. B. J.). Of all abstracts, duplicates were removed and twentythree added from other sources. In all, seventy-five full-text articles were retrieved, and thirty-six met all inclusion criteria and were included in the present review (Fig. 1). A data extraction form was developed, based on the PRISMA statement for reporting systematic reviews ${ }^{(18)}$. Data extraction was conducted by two authors (P. B. J. and E. V. C.) and included information about the article (e.g. authors, year), participants (e.g. demographics, BMI), study design, intervention characteristics (e.g. aim, length, follow-up, arms), outcome measures and main results.

The articles were grouped by study design as RCT (Table 1), randomised trials (RT, Table 2) and non-randomised trials (NRT, Table 3), whereas in the results text, articles were further presented by intervention type: diet-only, exercise-only and combined diet and exercise. Across studies, heterogeneity was observed in 
various parameters, including (i) study characteristics (sample size, completion rate, trial length, with or without behavioural intervention, methodology for NEPA/NEAT); (ii) participant characteristics (sex, age, BMI, ethnicity, activity level); (iii) diet (degree of energy restriction) or exercise prescriptions (mode, frequency, intensity, duration); (iv) assessment of NEPA/NEAT (ACC, heart rate (HR), activity diary, indirect calorimetry, DLW); and (v) main outcomes (compensation or non-compensation in NEPA and related energy expenditure, NEAT). If the outcome measure was PA assessed through activity monitors, then NEPA was used. If the outcome was non-exercise EE measured using DLW or assessment from accelerometry or other methods, NEAT was used. When PAEE was referred to as NEAT, an assumption that volitional exercise during the intervention was not performed was made. This terminology was used consistently throughout the manuscript to adequately differentiate these two concepts/outcomes. Considering this heterogeneity, a meta-analysis was found inappropriate. Results based on the extracted data were instead synthesised and presented grouped by study design (Tables 1-3) and intervention type (in the text).

Frequencies, medians, range and proportions were assessed using SPSS (version 24; IBM SPSS Statistics for Windows).

\section{Quality assessment}

Study quality was assessed with the Quality Assessment Tool for Quantitative Studies ${ }^{(52)}$ (online Supplementary material SII), evaluating six key methodological domains: study design, blinding, representativeness (selection bias), representativeness (withdrawals/dropouts), confounders and data collection. Each domain was classified as strong, moderate or weak methodological quality. A global rating was determined based on the scores of each component. Two authors independently rated the six domains and overall quality (P. B. J., E. V. C.). Discrepancies were discussed until a consensus was reached. Inter-rater agreement was good (Cohen's $\kappa=0 \cdot 68$ ). Quality assessment of all studies included in the review is provided as the online Supplementary material SIII.

\section{Results}

The initial search identified 1412 (1389 citations identified by database search and twenty-three through other sources) unique records, of which 314 were removed owing to duplication. From the remaining 1098 records, 1023 citations were excluded based on the screening of titles and abstracts. Full-text articles for the remaining seventy citations were retrieved and reviewed. A total of thirty-nine articles did not satisfy the inclusion criteria and were excluded; thus, thirty-six articles were considered (Fig. 1).

A total of thirty-six articles (10 (28\%) RCT, 9 (25\%) RT and 17 (47\%) NRT) with a total of seventy intervention arms (diet, exercise, diet plus exercise), comprising 1561 participants, met the inclusion criteria.

Behavioural compensation in NEPA or related decreases in NEAT were observed in twenty-six out of seventy intervention arms (fifteen out of thirty-six studies), whereas the remaining forty-four showed no compensation. From those who

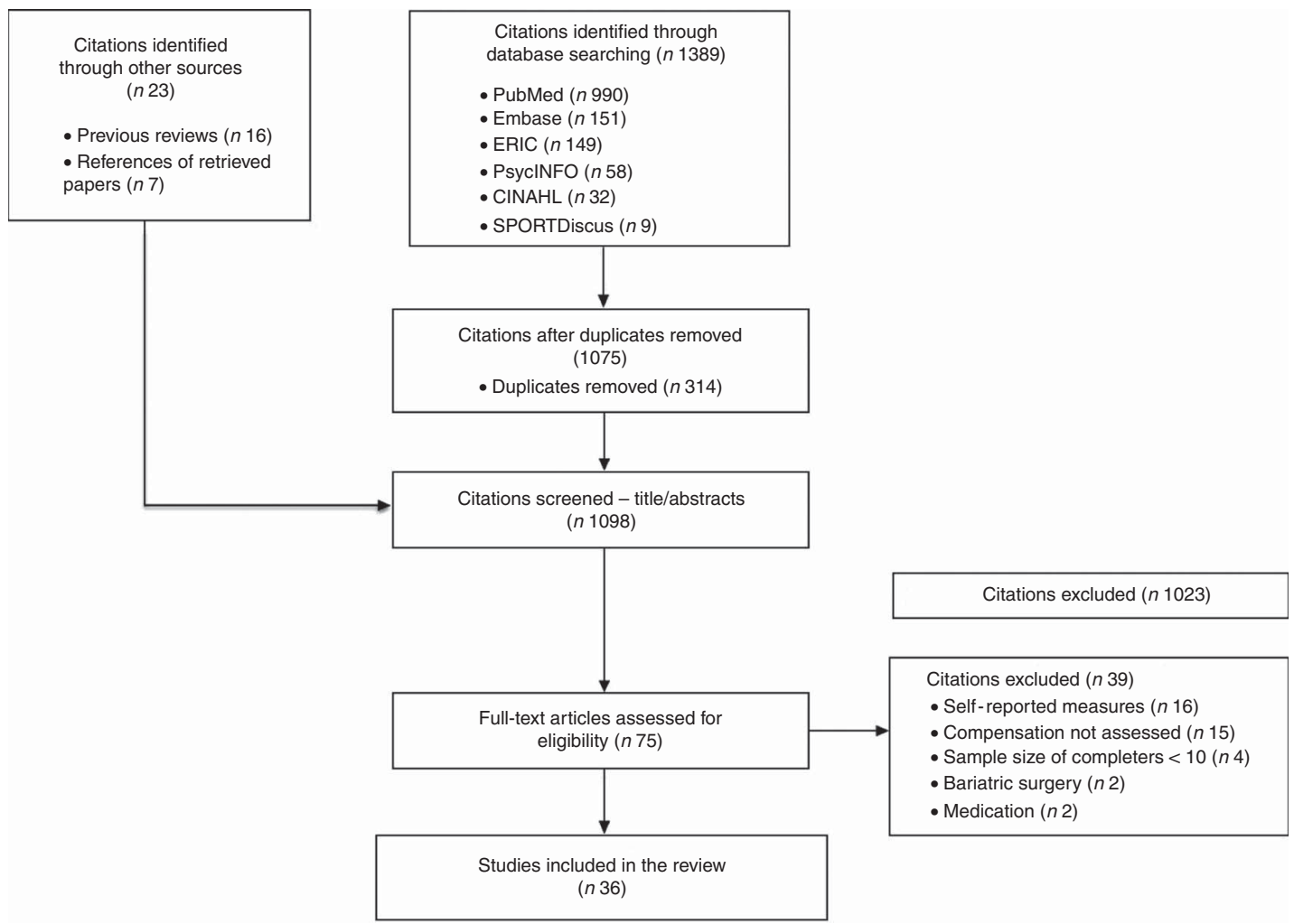

Fig. 1. Flow diagram. 
compensated (fifteen studies), fifteen intervention arms were diet-only interventions, eight were exercise-only interventions and three were diet plus exercise interventions.

Detailed information about the included studies is presented in Tables $1-3$, divided by design type - that is NRT, RT and RCT.

Studies will be further detailed by intervention type (diet-only, exercise-only and combined diet and exercise), as follows.

Intervention arms that decreased NEAT (i.e. twenty-one arms) presented a higher median value of weight loss (available in eighteen intervention arms: average of $-10 \mathrm{~kg}$ ) compared with those who showed no changes (available in thirty-eight intervention arms; average of $-5 \mathrm{~kg}$ ). Similar medians were observed for trial length, BMI and age. A similar trend was found when observing weight-loss medians for diet-only, exercise-only and combined diet and exercise, with higher weight loss found in the groups that reduced NEAT. In studies that showed reductions in NEPA, similar median weight loss, BMI, trials length and age were observed compared with those studies that reported no changes in NEPA. However, in dietonly interventions, weight loss observed in participants who decreased NEPA was double the weight loss found in those who did not compensate. The median study length of exerciseonly studies that showed decreases in NEAT was half the median length of trials that present no changes in NEAT. Compared with exercise-only studies without changes in NEAT or NEPA, the median exercise frequency was half in studies that showed reductions in NEAT, whereas the median exercise duration was double in trials that decreased NEPA. Studies with or without behavioural intervention had similar proportion of cases between compensators and non-compensator groups. In exercise-only and combined diet and exercise, studies with prescribed strength exercise are absent of cases with behavioural compensation (Table 4).

\section{Diet-only interventions}

The twenty-four diet-only interventions arms (i.e. fourteen dietonly trials) comprised approximately $39 \%$ of the total number of studies included in this review, with a total of five NRT (36\%), six RT (43\%) and three RCT (21\%).

\section{Study characteristics}

Sample size. Diet-only interventions comprised a total of 400 participants with a median sample size of 18 (range 5-66). NRT included a median sample size of 23 (range 6-66), RT of 17 (range 5-57) and RCT of 15 (range 15-33).

Completion rate. Compliance to prescribed diet was only reported by DeLany et al. ${ }^{(28)}$ as $55 \%$ and by Wang et $a l .{ }^{(33)}$ as $100 \%$. Since Leibel et $a l .{ }^{(43)}$ performed a laboratory-based study, full compliance with protocol was achieved. The remaining trials did not report compliance.

Trial length. The median length of the studies was 5.6 (range 2-12) months, varying from 3.5 (range 2-6) for NRT, 4 (range 2-6) for RT and 8 (range 6-12) for RCT.

Behavioural intervention. A total of five studies included behavioural therapy ${ }^{(14-16,22,35)}$ comprising $36 \%$ of the diet-only studies included in this review.
Energy restriction. EI was restricted by $25^{(15,22)}, 10,20,25$ and $30 \%^{(16)}, 33 \%^{(35)}, 51 \%$ of weight maintenance ${ }^{(37)}$ and $75 \%$ of resting $\mathrm{EE}(\mathrm{REE})^{(14)}$. EI was prescribed as $3724 \mathrm{~kJ} / \mathrm{d}^{(15,22)}$, approximately $2092 \mathrm{~kJ} / \mathrm{d}$ in the first 4 weeks followed by 4 weeks at approximately $3515 \mathrm{~kJ} / \mathrm{d}^{(29)}, 3347 \mathrm{~kJ} / \mathrm{d}^{(51)}$, approximately $2929 \mathrm{~kJ} / \mathrm{d}$ in the first 4 weeks and approximately $3347 \mathrm{~kJ} / \mathrm{d}$ in the next 8 weeks ${ }^{(49)}$, and according to body weight $(<90.7 \mathrm{~kg}, \quad 5021-6276 \mathrm{~kJ} / \mathrm{d} ;>90.7 \mathrm{~kg}$ and $<113.4 \mathrm{~kg}, \quad 6276-$ $7531 \mathrm{~kJ} / \mathrm{d}$; and $>113.4 \mathrm{~kg}, 7531-8368 \mathrm{~kJ} / \mathrm{d}^{(28)}$. EI was also prescribed as a reduction of $2929 \mathrm{~kJ} / \mathrm{d}^{(34)}, 3347 \mathrm{~kJ} / \mathrm{d}^{(43)}$ and $1682 \mathrm{~kJ} / \mathrm{d}^{(33)}$. EI was not reported in one study ${ }^{(27)}$.

\section{Participant characteristics}

Age. The median age across the fourteen studies was 40.5 years (range $25 \cdot 0-58 \cdot 6$ ), with values of 35.5 years (range 25-51) for NRT, 46.3 years (range 36.6-58.6) for RT and $39 \cdot 2$ years (range $34 \cdot 7-55 \cdot 2$ ) for RCT.

Sex. Seven studies included women only ${ }^{(14,27,29,33,37,49,51)}$, one study included men only ${ }^{(34)}$ and six studies included a combined sample of women and men ${ }^{(15,16,22,28,35,43)}$.

BMI. Four studies included overweight/obese individuals ${ }^{(33,35,43,49)}$. Non-obese individuals were included in four studies $^{(15,16,22,37,51)}$. Obese-only individuals were included in five studies $^{(14,27-29,34)}$. In the studies that provided data on this parameter, BMI was $31.2 \mathrm{~kg} / \mathrm{m}^{2}$ (range $27.4-43.6$ ), with a median of $31.4 \mathrm{~kg} / \mathrm{m}^{2}$ (range $28 \cdot 1-38.3$ ) for NRT, $35.0 \mathrm{~kg} / \mathrm{m}^{2}$ (range $31.7-$ 43.6) for RT and $27 \cdot 8 \mathrm{~kg} / \mathrm{m}^{2}$ (range $27 \cdot 4-27.9$ ) for RCT.

Ethnicity. Two studies described ethnic groups as Caucasian, Black, Asian and Hispanics ${ }^{(15,22)}$, and four studies reported participants as Caucasian and Black $^{(27,28,33,51)}$. One study included Caucasian only ${ }^{(37)}$ and seven studies did not report ethnic groups ${ }^{(14,16,29,34,35,43,49)}$.

Physical activity level. Only six studies characterised the level of PA of the participants as sedentary ${ }^{(14-16,22,33,35)}$.

Methods for assessing non-exercise activity thermogenesis. Among RCT, Martin et $a l^{(16)}$ assessed NEAT by subtracting the sum of REE from indirect calorimetry and thermic effect of food (TEF) (assumed as 0.1 TEE) from TEE by DLW. Redman et al. ${ }^{(15)}$ assessed NEAT (referred to as activity-related EE) as the residual value of the regression between measured TEE obtained from DLW and measured sleeping metabolic rate (SMR) using indirect calorimetry. In RT, DeLany et al. ${ }^{(28)}$ assessed NEAT (referred to as PAEE, as exercise was not prescribed) as TEE from DLW minus the sum of REE by indirect calorimetry with TEF (assumed as 0.1TEE). Kempen et al. ${ }^{(29)}$ assessed NEAT (referred to as PAEE, as exercise was not prescribed) by subtracting the sum of SMR from indirect calorimetry and the TEF (assumed as 0.1TEE) from TEE by DLW. Racette et al. ${ }^{(14)}$ assessed NEAT (referred to as PAEE, as exercise was not prescribed) with DLW for TEE, indirect calorimetry for REE and TEF as TEE - (REE + TEF). Wang et al. ${ }^{(33)}$ used ACC for nonexercise PAEE. Weigle ${ }^{(34)}$ used a $24-\mathrm{h}$ EE in a metabolic ward to assess NEAT (referred to as non-resting $\mathrm{EE}=24 \mathrm{EE}-\mathrm{REE}$ ). In NRT, Leibel et $a l^{(43)}$ assessed NEAT (referred to as non-resting EE) calculated as TEE from DLW minus the sum of REE and TEF obtained using a respiratory chamber. Weinsier et al. ${ }^{(51)}$ determine NEAT (referred to as PAEE, as exercise was not 
Table 1. Randomised controlled trials (ten studies)

\begin{tabular}{|c|c|c|c|c|c|c|}
\hline Studies & Sample & Intervention & Length + follow-up & Measures & Results & $\begin{array}{l}\text { Behavioural } \\
\text { response }\end{array}$ \\
\hline Church et al..$^{(19)}$ & $\begin{array}{l}\text { Control: } 94 \text { women } \\
\text { Age }=57(6) \\
\text { BMI }=32(4) \\
\text { Exercise-only interventions: } \\
\text { (a) } 139 \text { women } \\
\text { Age }=58(7) \\
\text { BMI }=31(4) \\
\text { (b) } 85 \text { women } \\
\text { Age }=57(6) \\
\text { BMl }=32(4) \\
\text { (c) } 93 \text { women } \\
\text { Age }=56(6) \\
\text { BMI }=31 \text { (4) }\end{array}$ & $\begin{array}{l}\text { Control: no intervention } \\
\text { Exercise-only interventions: } 3-4 \text { supervised } \\
\text { sessions per week at } 50 \% \mathrm{VO}_{2 \text { max. In week } 1 \text {, }} \\
\text { all exercisers expended } 17 \mathrm{~kJ} / \mathrm{kg} \text { per week } \\
\text { (KKW). Thereafter: } \\
\text { (a) This group remained at } 17 \mathrm{KKW} \\
\text { (b) This group increased to } 33 \mathrm{KKW} \\
\text { (c) This group increased to } 50 \mathrm{KKW} \\
\text { Training sessions alternated between recumbent } \\
\text { cycle-ergometer and treadmill }\end{array}$ & 6 Months & $\begin{array}{l}\text { Participants wore a pedometer daily except } \\
\text { when exercising }\end{array}$ & $\begin{array}{l}\text { Monitoring of steps per day indicated that } \\
\text { outside physical activity remained } \\
\text { constant throughout the trial for all } \\
\text { exercise groups } \\
\text { No significant differences were found } \\
\text { between groups at } 6 \text { months } \\
\text { In the } 17 \text { and } 33 \mathrm{KKW} \text { groups the actual } \\
\text { weight loss closely matched the } \\
\text { predicted weight loss. In the } 50 \mathrm{KKW} \\
\text { group the actual weight loss was lower } \\
\text { than predicted }\end{array}$ & $\begin{array}{l}\text { No compensation: } \\
\text { no change in } \\
\text { NEPA }\end{array}$ \\
\hline $\begin{array}{l}\text { Hollowell } \\
\text { et al. }\end{array}$ & $\begin{array}{l}\text { Control: } 4 \text { women; } 4 \text { men } \\
\text { Age }=51 \text { (7) } \\
\text { BMl }=31(3) \\
\text { Exercise-only interventions: } \\
\text { (a) Low amount/moderate } \\
\text { intensity: } 5 \text { women; } 3 \text { men } \\
\text { Age }=57 \text { (6) } \\
\text { BMl }=29 \text { (3) } \\
\text { (b) Low amount/vigorous } \\
\text { intensity: } 10 \text { women; } 10 \text { men } \\
\text { Age }=54(6) \\
\text { BMl }=30 \text { (3) } \\
\text { (c) High amount/vigorous } \\
\text { intensity: } 5 \text { women; } 9 \text { men } \\
\text { Age }=51 \text { (6) } \\
\text { BMl }=30 \text { (2) }\end{array}$ & $\begin{array}{l}\text { Control: no intervention } \\
\text { Exercise-only interventions: } 3-5 \text { times per } \\
\text { week } \\
\text { (a) Low amount/moderate intensity: } \\
40-55 \% \mathrm{VO}_{2} \text { peak to achieve EE of } \\
5029 \mathrm{~kJ} / \text { week } \\
\text { (b) Low amount/ vigorous intensity: } 65-85 \% \\
\mathrm{VO}_{2} \text { peak to achieve EE of } 5029 \mathrm{~kJ} / \mathrm{week} \\
\text { (c) High amount/vigorous intensity: } 65-85 \% \\
\mathrm{VO}_{2} \text { peak to achieve EE of } 8381 \mathrm{~kJ} / \text { week }\end{array}$ & 8 Months & $\begin{array}{l}\text { RT3 accelerometer (Stayhealthy, Inc.) worn for } \\
7 \mathrm{~d} \text { at baseline and end of intervention } \\
\text { Only days with } 9720 \text { total minutes of data in a } \\
24-\mathrm{h} \text { period included } \\
\text { All minute records from the RT3 file } \\
\text { corresponding to the prescribed exercise } \\
\text { were eliminated. The } 30 \text { min of RT3 data } \\
\text { immediately before and after exercise were } \\
\text { also eliminated }\end{array}$ & $\begin{array}{l}\text { The high-amount group showed a } \\
\text { significantly greater increase in non- } \\
\text { exercise PAEE } v \text {. controls } \\
\text { Non-exercise PAEE increased with } \\
\text { increasing exercise volume. } \\
\text { There was no significant difference in } \\
\text { change of non-exercise PAEE among } \\
\text { groups. } \\
\text { Change in body weight was not reported }\end{array}$ & $\begin{array}{l}\text { No compensation: } \\
\text { no change in } \\
\text { NEAT }\end{array}$ \\
\hline $\begin{array}{l}\text { Kozey-Keadle } \\
\text { et al. }{ }^{(21)}\end{array}$ & $\begin{array}{l}39 \text { women; } 19 \text { men } \\
\text { Control: } 10 \text { participants } \\
\text { Age }=43(10) \\
\text { BMI }=35(5) \\
\text { Interventions: } \\
\text { (a) Exercise (EX): } 16 \\
\text { participants } \\
\text { Age }=44(10) \\
\text { BMI }=35(5) \\
\text { Reduce Sedentary time (rST): } \\
14 \text { participants } \\
\text { Age }=45(10) \\
\text { BMI }=35(4) \\
\text { EX+rST: } 16 \text { participants } \\
\text { Age }=42(11) \\
\text { BMI }=35(4)\end{array}$ & $\begin{array}{l}\text { Control: maintain habitual behaviour } \\
\text { Interventions: } \\
\text { EX: Supervised aerobic exercise, } 5 \mathrm{~d} / \text { week, } \\
\text { at a moderate intensity ( } 40-65 \% \mathrm{VO}_{2} \text { peak), } \\
30-40 \text { min } \\
\text { rST: Strategies to increase NEPA and } \\
\text { pedometer with weekly goal provided. } \\
\text { Benefits of reducing ST discussed } \\
\text { EX + rST: combination of EX and rST }\end{array}$ & 12 Weeks & $\begin{array}{l}\text { Active PAL worn for a } 7-d \text { period at weeks } 3,6 \text {, } \\
9 \text { and } 12 \text { of the intervention }\end{array}$ & $\begin{array}{l}\text { For the EX group, ST did not decrease } \\
\text { significantly and NEPA did not change } \\
\text { from baseline to week } 12 \\
\text { Yet, the changes were variable, with } \\
\text { approximately } 50 \% \text { of participants } \\
\text { increasing ST and decreasing NEPA } \\
\text { The rST group decreased ST and } \\
\text { increased NEPA as expected } \\
\text { EX-rST significantly decreased ST and } \\
\text { increased time in NEPA from baseline to } \\
\text { week } 12 \\
\text { The control group increased ST } \\
\text { Body weight changes not reported }\end{array}$ & $\begin{array}{l}\text { No overall } \\
\text { compensation: no } \\
\text { change in NEPA }\end{array}$ \\
\hline Martin et al. ${ }^{(22)}$ & $\begin{array}{l}46 \text { (26 women; } 20 \text { men) non- } \\
\text { obese participants } \\
\text { Age }=37(2) \\
\mathrm{BMI}=28(0.4)\end{array}$ & $\begin{array}{l}\text { Control: healthy weight maintenance diet } \\
\text { matching } 100 \% \text { of energy requirements } \\
\text { Interventions: } \\
\text { (a) LCD: low-energy diet to achieve } 15 \% \\
\text { reduction in body mass ( } 3724 \mathrm{~kJ} / \mathrm{d} \text { ) } \\
\text { (b) CR: } 25 \% \text { energy restriction from } \\
\text { baseline energy requirements } \\
\text { (c) CR }+\mathrm{EX}: 12.5 \% \mathrm{CR}+12.5 \% \text { increase in } \\
\text { EE by structured aerobic exercise, } 5 \mathrm{~d} / \text { week }\end{array}$ & 6 Months & $\begin{array}{l}\text { SPA was measured in a metabolic chamber } \\
\text { TEE was measured by DLW } \\
\text { SMR was assessed by a metabolic cart }\end{array}$ & $\begin{array}{l}\text { SPA did not decrease in any groups from } \\
\text { baseline to month } 6 \text {, not even when } \\
\text { dieting groups were combined and } \\
\text { compared with controls } \\
\text { Percent activity in a respiratory chamber } \\
\text { and PAL did not change from baseline to } \\
\text { month } 6 \\
\text { All groups lost weight throughout the } \\
\text { intervention period (marginal in the } \\
\text { controls) }\end{array}$ & $\begin{array}{l}\text { No compensation: } \\
\text { no change in } \\
\text { NEPA }\end{array}$ \\
\hline Martin et al. ${ }^{(16)}$ & $\begin{array}{l}3 \text { sites-study } \\
\text { PBRC: } 19 \text { women; } 15 \text { men; } \\
\text { Control }(n 11) ; \text { CR-25\% ( } n \text { 12); } \\
\text { LCD }(n 11) \\
\text { Age }=39 \text { (7) } \\
\text { BMI }=28 \text { (2) }\end{array}$ & $\begin{array}{l}\text { Control: healthy weight maintenance diet } \\
\text { matching } 100 \% \text { of energy requirements } \\
\text { Diet-only interventions: } \\
\text { PBRC } \\
\text { (a) CR- } 25 \%: 25 \% \text { energy restriction from } \\
\text { baseline energy requirements }\end{array}$ & $\begin{array}{l}\text { PBRC: } 6 \text { months } \\
\text { TUFTS and WUSM: } \\
12 \text { months }\end{array}$ & $\begin{array}{l}\text { DLW over } 14 \mathrm{~d} \text { at baseline, } 3 \text { and } 6 \text { months } \\
\text { (PBRC), and } 3,6,9 \text { and } 12 \text { months (TUFTS } \\
\text { and WUSM) } \\
\text { Indirect calorimeter using a metabolic cart to } \\
\text { measure REE } \\
\text { PAEE = TEE - (REE + 0.1TEE) }\end{array}$ & $\begin{array}{l}\text { PBRC: significant decreases in PAEE and } \\
\text { PAL observed in the CR and LCD } \\
\text { groups at months } 3 \text { and } 6 \\
\text { Accelerometry data suggest that participants } \\
\text { decreased time spent in higher-intensity } \\
\text { activity in favour of lower-intensity activity }\end{array}$ & $\begin{array}{l}\text { Compensation: } \\
\text { reduction in NEAT } \\
\text { No compensation: no } \\
\text { change in NEPA }\end{array}$ \\
\hline
\end{tabular}




\section{N British Journal of Nutrition}

Table 1. Continued

\begin{tabular}{|c|c|c|c|c|c|c|}
\hline Studies & Sample & Intervention & Length + follow-up & Measures & Results & $\begin{array}{l}\text { Behavioural } \\
\text { response }\end{array}$ \\
\hline & 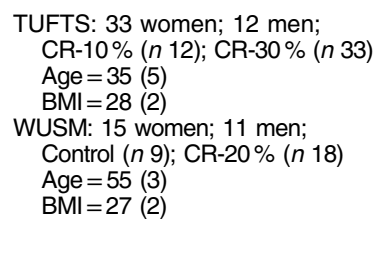 & $\begin{array}{l}\text { (b) LCD: low-energy diet to achieve } 15 \% \\
\text { reduction in body mass ( } 3724 \mathrm{~kJ} / \mathrm{d} \text { ) } \\
\text { TUFTS } \\
\text { (a) CR-10\%: } 10 \% \text { energy restriction from } \\
\text { baseline energy requirements } \\
\text { (b) CR-30\%: } 30 \% \text { energy restriction from } \\
\text { baseline energy requirements } \\
\text { WUSM } \\
\text { (a) CR-20\%: } 20 \% \text { energy restriction from } \\
\text { baseline energy requirements }\end{array}$ & & $\begin{array}{l}\text { Accelerometers: } \\
\text { Model } 7164 \text { (Actigraph); RT3 accelerometer } \\
\text { (Stayhealthy, Inc.) used at the same periods }\end{array}$ & $\begin{array}{l}\text { Significant weight loss in both groups. } \\
\text { TUFTS: significant decreases in PAEE and } \\
\text { PAL observed in both CR groups at } \\
\text { months } 6,9 \text { and } 12 \text {. No significant weight } \\
\text { loss in both groups } \\
\text { WUSM: significant change in PAEE only in } \\
\text { the CR group at month } 6 \text {. Significant } \\
\text { weight loss in both groups } \\
\text { Greater weight loss was associated with } \\
\text { larger decreases in PAEE }\end{array}$ & \\
\hline $\begin{array}{l}\text { Redman } \\
\text { et al. }{ }^{(15)}\end{array}$ & $\begin{array}{l}46 \text { participants (women and men) } \\
\text { Age }=37 \text { (1) } \\
\text { BMl }=28(1) \\
\text { Control: } 11 \text { participants } \\
\text { Interventions: } \\
\text { (a) Low-energy diet group } \\
\text { (LCD): } 11 \text { participants } \\
\text { (b) Energy restriction group } \\
\text { (CR): } 12 \text { participants } \\
\text { (c) CR + exercise (EX) group: } \\
12 \text { participants }\end{array}$ & $\begin{array}{l}\text { Control: healthy weight maintenance diet } \\
\text { matching } 100 \% \text { of energy requirements } \\
\text { Interventions: } \\
\text { (a) LCD: low-energy diet to achieve } 15 \% \\
\text { reduction in body mass ( } 3724 \mathrm{~kJ} / \mathrm{d}) \\
\text { (b) CR: } 25 \% \text { energy restriction from } \\
\text { baseline energy requirements } \\
\text { (c) CR + EX: } 12.5 \% \text { CR plus } 12.5 \% \\
\text { increase in energy expenditure by structured } \\
\text { aerobic exercise, } 5 \mathrm{~d} / \text { week }\end{array}$ & 6 Months & $\begin{array}{l}\text { DLW over } 14 \mathrm{~d} \text { at baseline, weeks } 10-12 \text { and } \\
\text { weeks } 22-24 \\
\text { SMR by metabolic chamber } \\
\text { Activity-related EE calculated as the residual } \\
\text { value of the regression between measured } \\
\text { TEE and SMR }\end{array}$ & $\begin{array}{l}\text { Activity-related EE decreased throughout } \\
\text { the intervention period in LCD and CR, } \\
\text { but not in CR+EX or controls } \\
\text { Body weight decreased in all groups from } \\
\text { baseline to } 6 \text { months }\end{array}$ & $\begin{array}{l}\text { Compensation: } \\
\text { NEAT decreased } \\
\text { in the diet-only } \\
\text { groups }\end{array}$ \\
\hline $\begin{array}{l}\text { Rosenkilde } \\
\text { et al. }{ }^{(23)}\end{array}$ & $\begin{array}{l}\text { Control: } 17 \text { men } \\
\text { Age }=31(6) \\
\text { BMl }=28(3) \\
\text { Exercise-only interventions: } \\
\text { (a) MOD: } 18 \text { men } \\
\text { Age }=30(7) \\
\text { BMl }=29(2) \\
\text { (b) HIGH: } 18 \text { men } \\
\text { Age }=28(5) \\
\text { BMl }=28(1)\end{array}$ & $\begin{array}{l}\text { Control: no intervention } \\
\text { Exercise-only interventions: } \\
\text { (a) MODERATE: Aerobic exercise (e.g. } \\
\text { running, cycling) at moderate intensity, } \\
30-60 \mathrm{~min}, 3 \mathrm{~d} / \mathrm{week}(1255 \mathrm{~kJ} / \mathrm{d}) \\
\text { (b) HIGH: Aerobic exercise (e.g. running, } \\
\text { cycling) at high intensity, 30-60 min, } \\
3 \mathrm{~d} / \mathrm{week}(2510 \mathrm{~kJ} / \mathrm{d})\end{array}$ & 13 Weeks & $\begin{array}{l}\text { Model GT1M (Actigraph) worn for } 3 \mathrm{~d} \text {, at } \\
\text { baseline and at } 6 \text { and } 11 \text { weeks after } \\
\text { randomisation } \\
\text { Non-exercise PA was obtained by subtracting } \\
\text { activity counts during prescribed exercise } \\
\text { from total activity counts }\end{array}$ & $\begin{array}{l}\text { When the exercise component was } \\
\text { subtracted from total activity counts, no } \\
\text { significant difference in NEPA was found } \\
\text { between any of the groups } \\
\text { Body weight decreased similarly in both } \\
\text { exercise groups }\end{array}$ & $\begin{array}{l}\text { No compensation: } \\
\text { no change in } \\
\text { NEPA }\end{array}$ \\
\hline Turner et al. ${ }^{(24)}$ & $\begin{array}{l}\text { Control: } 14 \text { men } \\
\text { Age }=53(4) \\
\text { BMl }=28(3) \\
\text { Exercise-only intervention: } \\
15 \text { men } \\
\mathrm{Age}=55(5) \\
\mathrm{BMI}=28(3)\end{array}$ & $\begin{array}{l}\text { Control: no intervention } \\
\text { Exercise-only intervention: Walking, running, } \\
\text { cycling progressing from } 30 \mathrm{~min}, 3 \mathrm{~d} / \text { week, } \\
50 \% \mathrm{VO}_{2 \text { max }} \text { at weeks } 1-2 \text { to } 60 \mathrm{~min}, \\
4 \mathrm{~d} / \text { week, } 70 \% \mathrm{VO}_{2 \max } \text { by week } 24 ; \\
\text { supervised sessions approximately } 10 \%\end{array}$ & 24 Weeks & $\begin{array}{l}\text { Actiheart (CamNtech Ltd) (combined HR and } \\
\text { accelerometer) worn over } 7 \mathrm{~d} \text {, at baseline, } \\
\text { weeks 2, } 9 \text { and } 18 \text { and at } 2 \text { weeks in } \\
\text { detraining. } \\
\text { Non-prescribed PAEE was calculated by } \\
\text { subtracting prescribed PAEE from overall } \\
\text { PAEE }\end{array}$ & $\begin{array}{l}\text { Prescribed exercise had no detrimental } \\
\text { effect on time spent in non-prescribed } \\
\text { PA and PAEE } \\
\text { There was a trend for greater non- } \\
\text { prescribed PAEE in the exercise group } \\
\text { and no change in the controls. } \\
\text { Significant change in body mass only in the } \\
\text { exercisers }\end{array}$ & $\begin{array}{l}\text { No compensation: } \\
\text { no change in } \\
\text { NEAT or NEPA }\end{array}$ \\
\hline $\begin{array}{l}\text { Whybrow } \\
\text { et al. }\end{array}$ & $\begin{array}{l}6 \text { men } \\
\mathrm{Age}=30(6) \\
\mathrm{BMl}=24(2) \\
6 \text { women } \\
\mathrm{Age}=25(6) \\
\mathrm{BMl}=23(2)\end{array}$ & $\begin{array}{l}\text { Cross-over design with: } \\
\text { Control phase: no additional exercise } \\
\text { Exercise-only phases: } \\
\text { (a) MOD, two } 40 \text {-min sessions, in cycle } \\
\text { ergometer, to expend } 28.6 \mathrm{~kJ} / \mathrm{kg} \\
\text { (b) HIGH, three } 40 \text {-min sessions, cycle } \\
\text { ergometer, to expend } 57.1 \mathrm{~kJ} / \mathrm{kg}\end{array}$ & $14 d$ & $\begin{array}{l}\text { TEE was assessed by DLW over } 14 \mathrm{~d} \\
\text { Non-exercise } \mathrm{EE}=\mathrm{TEE}-\mathrm{ExEE}\end{array}$ & $\begin{array}{l}\text { Non-exercise EE was not significantly } \\
\text { different between study conditions } \\
\text { No significant changes in body weight in all } \\
\text { phases }\end{array}$ & $\begin{array}{l}\text { No compensation: } \\
\text { NEAT was not } \\
\text { affected by the } \\
\text { treatment } \\
\text { condition }\end{array}$ \\
\hline Willis et al. ${ }^{(26)}$ & $\begin{array}{l}\text { Control: } 9 \text { women; } 9 \text { men } \\
\text { Age }=23(3) \\
\text { BMl }=30(4) \\
\text { Exercise-only interventions: } \\
\text { (a) } 1674 \mathrm{~kJ}: \\
19 \text { women; } 18 \text { men } \\
\text { Age }=23(3) \\
\mathrm{BMl}=31(6) \\
\text { (b) } 2510 \mathrm{~kJ}: \\
18 \text { women; } 19 \text { men } \\
\text { Age }=23(3) \\
\text { BMl }=31(4)\end{array}$ & $\begin{array}{l}\text { Control: no intervention } \\
\text { Exercise-only interventions: } \\
\text { (a) Aerobic exercise training } 5 \mathrm{~d} / \text { week at } \\
1674 \mathrm{~kJ} / \text { session } \\
\text { (b) Aerobic exercise training } 5 \mathrm{~d} / \text { week at } \\
2510 \mathrm{~kJ} / \text { session }\end{array}$ & 10 Months & $\begin{array}{l}\text { REE was assessed by indirect calorimetry. } \\
\text { TEE was assessed using DLW over a 14-d } \\
\text { period. Both assessed at baseline and } \\
10 \text { months } \\
\text { Non-EXEE }=(0.9 T E E-R E E)-\text { net ExEE } \\
\text { (EXEE }- \text { REE) } \\
\text { NEPA and sedentary time were assessed by } \\
\text { an accelerometer (model GT1M; Actigraph) }\end{array}$ & $\begin{array}{l}\text { Within the exercise groups, there were no } \\
\text { significant effects of group, time or } \\
\text { group-time interaction for NEPA } \\
\text { No significant within- or between-group } \\
\text { differences in change in non-exercise EE } \\
\text { Activity counts were higher in the 2510-kJ } \\
\text { group } \\
\text { Body weight decreased in exercise groups } \\
\text { and slightly increased in controls }\end{array}$ & $\begin{array}{l}\text { No compensation: } \\
\text { no changes in } \\
\text { NEPA or NEAT }\end{array}$ \\
\hline
\end{tabular}

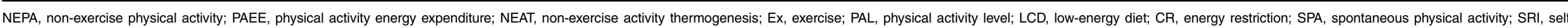

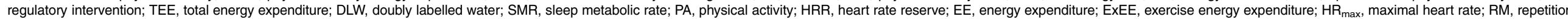

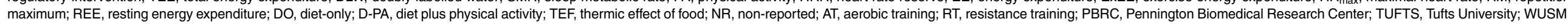
Washington University School of Medicine. 
Table 2. Randomised trials with no control group (nine studies)

\begin{tabular}{|c|c|c|c|c|c|c|}
\hline Studies & Sample & Intervention & Length + follow-up & Measures & Results & $\begin{array}{l}\text { Behavioural } \\
\text { response }\end{array}$ \\
\hline Brehm et al. ${ }^{(27)}$ & $\begin{array}{l}\text { Low-carbohydrate diet (low-carb): } \\
20 \text { women } \\
\mathrm{Age}=45(2) \\
\mathrm{BMI}=33(0.5) \\
\text { Energy-restricted, low-fat diet: } \\
20 \text { women } \\
\mathrm{Age}=41(3) \\
\mathrm{BMI}=34(0.5)\end{array}$ & $\begin{array}{l}\text { Low-carb: Ad libitum low-carb diet } \\
\text { Low-fat: energy-restricted, low-fat diet }\end{array}$ & 4 Months & Pedometer records (number of steps per day) & $\begin{array}{l}\text { Estimates of PA were stable in the dieters } \\
\text { during the study and did not differ } \\
\text { between groups } \\
\text { Both groups lost weight throughout the } \\
\text { intervention }\end{array}$ & $\begin{array}{l}\text { No compensation: } \\
\text { no changes in } \\
\text { NEPA }\end{array}$ \\
\hline $\begin{array}{l}\text { DeLany } \\
\text { et al. }\end{array}$ & $\begin{array}{l}116 \text { severely obese, } 87 \% \text { women, } \\
35 \% \text { African American } \\
\text { Age }=48(6) \\
\text { BMI }=44(5) \\
\text { DO: } 57 \text { participants } \\
\text { D-PA: } 61 \text { participants }\end{array}$ & $\begin{array}{l}\text { Diet alone (DO): prescribed energy restriction } \\
\text { of } 5021-6276 \mathrm{~kJ} / \mathrm{d} \text { for those weighing } \\
<90.7 \mathrm{~kg}, 6276-7531 \mathrm{~kJ} / \mathrm{d} \text { for those weight } \\
\geq 90.7 \text { but }<113.4 \mathrm{~kg} \text {, and } 7531-8786 \mathrm{~kJ} / \mathrm{d} \\
\text { for those weighing }>113.4 \mathrm{~kg} \\
\text { Diet plus PA (D-PA): prescribed energetic } \\
\text { restriction + moderate-intensity PA, similar to } \\
\text { brisk walking, prescribed and progressing to } \\
60 \mathrm{~min}, 5 \mathrm{~d} / \text { week }\end{array}$ & 12 Months & $\begin{array}{l}\text { TEE measured with DLW at baseline and } \\
12 \text { months; TEE assessed at baseline and } \\
6 \text { months using DLW } \\
\text { REE was measured at baseline, } 6 \text { and } \\
12 \text { months by indirect calorimetry } \\
\text { Multisensor physical activity monitors worn } \\
\text { during DLW periods and at } 12 \text { months } \\
\text { PAEE = TEE }- \text { (REE }+0.01 \times \text { TEE) (but ExEE } \\
\text { not determined) }\end{array}$ & $\begin{array}{l}\text { Although counselled to maintain PA levels, } \\
\text { over half of the DO group showed an } \\
\text { increase in steps } \\
40 \% \text { of the D-PA group showed an } \\
\text { increase of less than } 500 \text { steps } / \text {, and } \\
27 \% \text { showed no increase or non- } \\
\text { significant decrease } \\
\text { No significant change in PAEE between } \\
\text { groups }\end{array}$ & $\begin{array}{l}\text { No compensation: } \\
\text { no changes in } \\
\text { NEAT } \\
\text { NEPA increased in } \\
\text { the DO group }\end{array}$ \\
\hline $\begin{array}{l}\text { Kempen } \\
\text { et al. }\end{array}$ & $\begin{array}{l}\text { Diet: } 10 \text { women } \\
\text { Age }=37(2) \\
\text { BMI }=32(1) \\
\text { Diet + exercise: } 10 \text { women } \\
\text { Age }=39(5) \\
\text { BMI }=32(1)\end{array}$ & $\begin{array}{l}\text { Diet: } 4 \text { weeks of a low-energy diet } \\
\text { (approximately } 2092 \mathrm{~kJ} / \mathrm{d} \text { ); } 4 \text { weeks of a } \\
\text { mixed, balanced diet (approximately } \\
3515 \mathrm{~kJ} / \mathrm{d} \text { ) } \\
\text { Diet + exercise: } 90 \text { min sessions, } 3 \mathrm{~d} / \mathrm{week} \\
\text { aerobic dancing and fitness } \\
\text { (cardiac + strength training), at } 50-60 \% \\
\mathrm{VO}_{2 \max }\end{array}$ & 8 Weeks & $\begin{array}{l}\text { SMR measured on calorimeter } \\
\text { TEE assessed by DLW at baseline and weeks } \\
7-8 \\
\text { PAEE }=\text { TEE }-(\text { SMR }+0 \cdot 1 \mathrm{TEE} \text { ) (diet group) } \\
\mathrm{PAEE}=\mathrm{TEE}-(\mathrm{SMR}+0 \cdot 1 \mathrm{TEE}+\mathrm{ExEE}) \\
\text { (Ex+ diet group) } \\
\text { ExEE assessed by HR monitor }\end{array}$ & $\begin{array}{l}\text { TEE decreased in both groups } \\
\text { No significant differences in PAEE within } \\
\text { and between groups. } \\
\text { Energy expended during ExEE in Diet + Ex } \\
\text { group was compensated by reducing } \\
\text { non-exercise daily activities } \\
\text { Significant weight loss in both groups }\end{array}$ & $\begin{array}{l}\text { Compensation: } \\
\text { decrease in NEAT } \\
\text { in the diet plus } \\
\text { exercise arm } \\
\text { No compensation: no } \\
\text { change in NEAT in } \\
\text { the diet-only arm }\end{array}$ \\
\hline Nicklas et al. ${ }^{(30)}$ & $\begin{array}{l}\text { DIET + EX: } 15 \text { women; } 5 \text { men } \\
\text { Age }=70(3) \\
\text { BMI }=33(3) \\
\text { SRI+DIET + EX: } 16 \text { women; } \\
5 \text { men } \\
\text { Age }=71 \text { (4) } \\
\text { BMI }=34(2)\end{array}$ & $\begin{array}{l}\text { DIET + EX: hypoenergetic diet }(2510 \mathrm{~kJ} / \mathrm{d} \\
\text { deficit) and treadmill walking, } 4 \mathrm{~d} / \mathrm{week} \text {, at } \\
65-70 \% \text { HRR, progressing from } 15-20 \mathrm{~min} \\
\text { at } 50 \% \text { HRR at week } 1 \text { to } 30 \mathrm{~min} \text { at } 65-70 \% \\
\text { HRR by the end of the week } 6 \\
\text { SRI + DIET + EX: hypoenergetic diet } \\
\text { (-2510 kJ/d deficit) + exercise + self- } \\
\text { regulatory intervention (SRI) to promote SPA }\end{array}$ & $\begin{array}{l}5 \text { months } \\
\text { 5-month Follow-up }\end{array}$ & $\begin{array}{l}\text { SPA was measured by tri-axial accelerometer } \\
\text { worn for } 7 \mathrm{~d} \text { at baseline, } 5 \text { and } 10 \text { months, } \\
\text { and operationalised as minutes of light PA }\end{array}$ & $\begin{array}{l}\text { PA increased in both groups during the } \\
\text { weight-loss phase } \\
\text { Adjusted changes in minutes of light } \\
\text { activity tended to be significantly greater } \\
\text { in the SRI+DIET + EX group compared } \\
\text { with the DIET+EX group } \\
\text { Both groups reduced body weight during } \\
\text { the intervention and regained during } \\
\text { follow-up. Greater weight loss and lower } \\
\text { regain found in the SRI + DIET + EX } \\
\text { group }\end{array}$ & $\begin{array}{l}\text { No compensation: } \\
\text { NEPA increased }\end{array}$ \\
\hline $\begin{array}{l}\text { Racette } \\
\quad \text { et al. }\end{array}$ & $\begin{array}{l}\text { Low-fat non-exercise: } 7 \text { obese } \\
\text { women } \\
\text { Age }=37 \text { (4) } \\
\text { BMI }=\text { NR } \\
\text { Low-carb non-exercise: } 6 \text { obese } \\
\text { women } \\
\text { Age }=41(6) \\
\text { BMI }=\text { NR } \\
\text { Low-fat exercise: } 5 \text { obese women } \\
\text { Age }=40(4) \\
\text { BMI }=\text { NR } \\
\text { Low-carb exercise: } 5 \text { obese } \\
\text { women } \\
\text { Age }=36(5) \\
\text { BMI }=\text { NR }\end{array}$ & 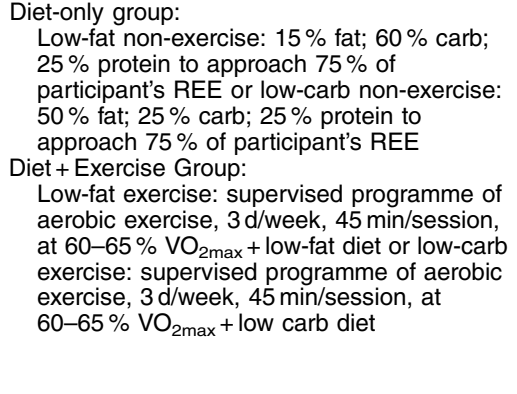 & 12 Weeks & $\begin{array}{l}\text { TEE by DLW over a 2-week period at baseline } \\
\text { and end of intervention } \\
\text { REE and TEF measured by indirect calorimetry } \\
\text { PAEE }=\text { TEE - (REE }+0.01 \times T E F) \\
\text { ExEE assessed by HR monitor } \\
\text { Heart rate monitor worn on } 3 \text { d during DLW } \\
\text { period ( } n \text { 14) }\end{array}$ & $\begin{array}{l}\text { The addition of aerobic exercise during the } \\
\text { reducing diet proved to be an effective } \\
\text { method for preventing the decrease in } \\
\text { PA. The non-exercise group decreased } \\
\text { PA and non-exercise energy } \\
\text { expenditure, and thus had } \\
\text { compensation } \\
\text { Significant reductions in body weight in all } \\
\text { groups }\end{array}$ & $\begin{array}{l}\text { Compensation: } \\
\text { NEPA and NEAT } \\
\text { decreased in the } \\
\text { diet-only group } \\
\text { No compensation: no } \\
\text { change in NEPA } \\
\text { and NEAT in the } \\
\text { diet plus exercise } \\
\text { group }\end{array}$ \\
\hline
\end{tabular}




\section{N British Journal of Nutrition}

Table 2. Continued

\begin{tabular}{|c|c|c|c|c|c|c|}
\hline Studies & Sample & Intervention & Length + follow-up & Measures & Results & $\begin{array}{l}\text { Behavioural } \\
\text { response }\end{array}$ \\
\hline $\begin{array}{l}\text { Rangan } \\
\text { et al. }\end{array}$ & $\begin{array}{l}\text { Aerobic training (AT): } 14 \text { women, } \\
14 \text { men. } \\
\text { Age }=52(9) \\
\text { BMl }=31(3) \\
\text { Resistance Training (RT): } \\
20 \text { women; } 8 \text { men. } \\
\text { Age }=51 \text { (12) } \\
\text { BMI }=30(3) \\
\text { AT/RT: } 16 \text { women; } 10 \text { men } \\
\text { Age }=48(11) \\
\text { BMl }=31(3)\end{array}$ & $\begin{array}{l}\text { AT: Treadmill, elliptical or cycle ergometer } \\
\text { exercise progressed over } 8-10 \text { weeks to } \\
\text { reach a volume of approximately } 7.4 \mathrm{~km} / \\
\text { week } \\
\text { RT: } 3 \mathrm{~d} / \text { week, } 3 \text { sets, } 8-12 \text { reps, } 8 \text { exercises for } \\
\text { major muscle groups } \\
\text { AT/RT: full combination of both exercise } \\
\text { prescriptions }\end{array}$ & 8 Months & $\begin{array}{l}\text { RT3 accelerometer (Stayhealthy, Inc.) worn for } \\
7 \mathrm{~d} \text { at baseline and end of intervention } \\
\text { All known exercise data were removed from the } \\
\text { raw accelerometer files. The } 30 \text { min of RT3 } \\
\text { data immediately before and after exercise } \\
\text { were also eliminated }\end{array}$ & $\begin{array}{l}\text { No significant change in non-exercise } \\
\text { PAEE in any of the exercise training } \\
\text { groups } \\
\text { Change in body weight not reported }\end{array}$ & $\begin{array}{l}\text { No compensation: } \\
\text { no change in } \\
\text { NEAT }\end{array}$ \\
\hline Schutz et al. ${ }^{(32)}$ & $\begin{array}{l}55 \text { normal-weight and overweight } \\
\text { women } \\
\text { Age }=27 \text { (1) } \\
\text { BMl }=25 \text { (5) }\end{array}$ & $\begin{array}{l}\text { Prescribing additional PA (walking only) of } \\
30 \mathrm{~min} / \mathrm{d} \\
\text { Prescribing additional PA (walking only) of } \\
60 \mathrm{~min} / \mathrm{d} \\
\text { Prescribing additional PA (walking only) of } \\
90 \mathrm{~min} / \mathrm{d}\end{array}$ & 8 Weeks & Uniaxial accelerometer to measure PA & $\begin{array}{l}\text { Compensation increased progressively as } \\
\text { length of prescription increased } \\
\text { The average compensation rate calculated } \\
\text { as the ratio between expected steps/d } \\
\text { (from baseline steps/d) by the observed } \\
\text { steps/d from prescribed walking was } \\
25 \% \text { with substantial inter-individual } \\
\text { variation }\end{array}$ & $\begin{array}{l}\text { Compensation: } \\
\text { decrease in NEPA } \\
\text { for the } 60-\text { and } 90- \\
\text { min walking arms }\end{array}$ \\
\hline Wang et al. ${ }^{(33)}$ & $\begin{array}{l}34 \text { women } \\
\text { Age }=50-70 \\
\text { BMI }=25-40 \\
\text { Diet }(n 11) \\
\text { Diet }+ \text { LO-EX }(n 12) \\
\text { Diet }+ \text { HI-EX } \\
\quad(n 11)\end{array}$ & $\begin{array}{l}\text { Diet: hypoenergetic diet (reduction of } \\
1682 \mathrm{~kJ} / \mathrm{d} \text { ) } \\
\text { Diet + LO-EX: hypoenergetic diet (reduction of } \\
1443 \mathrm{~kJ} / \mathrm{d} \text { ) + treadmill walking, } 3 \mathrm{~d} / \text { week, } \\
\text { progressing from } 15-20 \mathrm{~min} \text { at } 45-50 \% \text { of } \\
\text { HRR on week } 1 \text { to } 55 \mathrm{~min} \text { at } \\
45-50 \% \text { HRR } \\
\text { Diet + HI-EX: hypoenergetic diet (reduction of } \\
1443 \mathrm{~kJ} / \mathrm{d} \text { ) + treadmill walking, } 3 \mathrm{~d} / \mathrm{week}, \\
\text { progressing from } 15-20 \mathrm{~min} \text { at } 45-50 \% \text { of } \\
\text { HRR on week } 1 \text { to } 30 \mathrm{~min} \text { at } \\
70-75 \% \text { HRR }\end{array}$ & $\begin{array}{l}20 \text { weeks } \\
12 \text {-month follow-up }\end{array}$ & $\begin{array}{l}\text { REE measured via indirect calorimetry } \\
\text { PAEE measured by RT3 accelerometer } \\
\text { (Stayhealthy, Inc.) }\end{array}$ & $\begin{array}{l}\text { Decreases in non-exercise PAEE during } \\
\text { intervention } \\
\text { Significant reductions in body weight in all } \\
\text { groups } \\
\text { The amount of weight regained after } 6 \text { and } \\
12 \text { months was inversely associated with } \\
\text { decreases in PAEE }\end{array}$ & $\begin{array}{l}\text { Compensation: } \\
\text { NEAT decreased } \\
\text { in all the } \\
\text { intervention arms }\end{array}$ \\
\hline Weigle et al. ${ }^{(34)}$ & $\begin{array}{l}\text { Diet: } 5 \text { men } \\
\text { Age }=53(9) \\
\text { BMI }=37(3) \\
\text { Diet plus vest for weight } \\
\text { replacement: } 5 \text { men } \\
\text { Age }=49(8) \\
\text { BMI }=35(2)\end{array}$ & $\begin{array}{l}\text { Diet-only group received a } 2929-\mathrm{kJ} \text { liquid diet } \\
\text { Diet (2929-kJ liquid diet) plus a vest into which } \\
\text { weights were inserted twice weekly to } \\
\text { replace the weight lost }\end{array}$ & 17 weeks & $\begin{array}{l}\text { Waist motion sensor pedometer worn the } \\
\text { entire intervention period } \\
24-\mathrm{h} \text { in a metabolic ward }\end{array}$ & $\begin{array}{l}\text { PA declined slowly and equally in both } \\
\text { groups. } \\
\text { Significant weight loss in both groups }\end{array}$ & $\begin{array}{l}\text { Compensation: } \\
\text { decreases in } \\
\text { NEPA and NEAT } \\
\text { in both } \\
\text { intervention arms }\end{array}$ \\
\hline
\end{tabular}

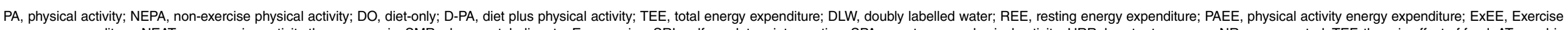

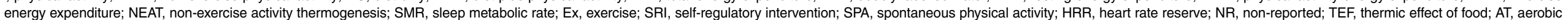
training; RT, resistance training; LO-Ex, low exercise; HI-Ex, high exercise. 
Table 3. Non-randomised trials (seventeen studies)

\begin{tabular}{|c|c|c|c|c|c|c|}
\hline Studies & Sample & Intervention & Length + follow-up & Measures & Results & Behavioural response \\
\hline $\begin{array}{l}\text { Bonomi } \\
\text { et al. }\end{array}$ & $\begin{array}{l}66 \text { overweight and obese } \\
\text { subjects: } 56 \text { women; } \\
10 \text { men } \\
\mathrm{Age}=51(12) \\
\mathrm{BMI}=38(7)\end{array}$ & Intervention consisted in $67 \%$ energy restriction & 12 Weeks & $\begin{array}{l}\text { PA was measured using a tri-axial } \\
\text { accelerometer }\end{array}$ & $\begin{array}{l}\text { Body weight decreased by } 14 \mathrm{~kg} \text { and activity } \\
\text { counts increased } \\
\text { Significantly less sedentary time and increased } \\
\text { time spent walking and bicycling were } \\
\text { observed } \\
\text { Significant reduction in body weight }\end{array}$ & $\begin{array}{l}\text { No compensation: } \\
\text { NEPA increased }\end{array}$ \\
\hline $\begin{array}{l}\text { Colley } \\
\quad \text { et al. }\end{array}$ & $\begin{array}{c}13 \text { obese women } \\
\mathrm{Age}=41(12) \\
\mathrm{BMI}=34(5)\end{array}$ & $\begin{array}{l}\text { Structured moderate-intensity walking } \\
\text { programme with a weekly target of } 6276 \mathrm{~kJ} / \\
\text { week. A 4-week supervised phase (including } \\
3-4 \text { sessions/week) was followed by a 4-week } \\
\text { unsupervised phase }\end{array}$ & 8 Weeks & $\begin{array}{l}\text { RT3 accelerometer (Stayhealthy, } \\
\text { Inc.) worn for } 14 \mathrm{~d} \text { at baseline } \\
\text { and weeks } 3-4 \\
\text { TEE by DLW used on } 7 \\
\text { participants at baseline and } \\
\text { weeks } 3-4 \\
\text { REE by indirect calorimetry } \\
\text { NEAT = TEE-(REE + } \\
0.1 \text { TEE + ExEE) }\end{array}$ & $\begin{array}{l}\text { Accelerometer data showed no change in the } \\
\text { time spent in sedentary, light or moderate } \\
\text { activity from baseline to the intervention } \\
\text { (week 4) } \\
\text { NEAT decreased } 22 \% \text { from baseline to the } \\
\text { intervention (week } 4 \text { ) } \\
\text { Change in body weight not reported }\end{array}$ & $\begin{array}{l}\text { Compensation: NEAT } \\
\text { decreased }\end{array}$ \\
\hline $\begin{array}{l}\text { De Groot } \\
\text { et al. }\end{array}$ & $\begin{array}{l}13 \text { overweight women (10 } \\
\text { re-evaluated at } \\
1 \text { month follow-up; } 8 \\
\text { re-evaluated at 1-year } \\
\text { Follow-up) } \\
\text { Age }=39 \text { (6) } \\
\mathrm{BMI}=28(2)\end{array}$ & $\begin{array}{l}\text { Slimming programme with low-energy diet } \\
\text { tailored to each individual energy requirement } \\
\text { ( } 51 \% \text { of weight maintenance intake), followed } \\
\text { by a weight maintenance diet, adjusted for } \\
\text { weight lost - prescribed until } 1 \text { year of } \\
\text { follow-up }\end{array}$ & $\begin{array}{l}8 \text { Weeks } \\
1 \text {-Year } \\
\text { follow-up }\end{array}$ & $\begin{array}{l}\text { Metabolic chamber used to } \\
\text { measure TEE and PAEE } \\
\text { Actometer and Doppler metre } \\
\text { counts }\end{array}$ & $\begin{array}{l}\text { No difference was found between TEE before } \\
\text { and } 1 \text { month after slimming } \\
\text { PA increased } 12 \% \text { during intervention but } \\
\text { remained below baseline PA. } 1 \text { year after, } \\
\text { reduced level of PA as in the } 1 \text {-month } \\
\text { follow-up was observed } \\
\text { Spontaneous PA decreased during slimming and } \\
\text { tended to increase afterwards }\end{array}$ & $\begin{array}{l}\text { Compensation: } \\
\text { reductions in NEPA } \\
\text { during the slimming } \\
\text { period }\end{array}$ \\
\hline $\begin{array}{l}\text { Di Blasio } \\
\text { et al. }\end{array}$ & $\begin{array}{l}34 \text { post-menopausal } \\
\text { women } \\
\begin{array}{c}\mathrm{Age}=56(4) \\
\mathrm{BMI}=27(4)\end{array}\end{array}$ & $\begin{array}{l}\text { Walking programme at moderate intensity, } 4 \mathrm{~d} / \\
\text { week, progressing from } 40 \text { min at RPE } 11 \text { (on } \\
15 \text {-category RPE scale) at month } 1 \text { to } \\
50 \text { min at RPE } 13 \text { at month } 3\end{array}$ & 13 Weeks & $\begin{array}{l}\text { SenseWear } \mathrm{PrO}_{2} \text { armband worn } \\
\text { for } 3 \text { consecutive days } \\
(2 \text { weekdays and } 1 \text { weekend } \\
\text { day), at baseline and end of } \\
\text { intervention } \\
\text { Measurements included both } \\
\text { training and non-training days }\end{array}$ & $\begin{array}{l}\text { No significant change in TEE or non-exercise } \\
\text { PAEE in the complete sample, but two } \\
\text { subgroups were identified: } 44 \% \text { showed } \\
\text { reduced non-ex PAEE and TEE of non-ex } \\
\text { training days (GROUP-, non-responders), } \\
\text { whereas } 56 \% \text { increased their non-ex PAEE } \\
\text { and TEE (GROUP+, responders) } \\
\text { Change in body weight not reported }\end{array}$ & $\begin{array}{l}\text { Compensation: } \\
\text { reduction in NEAT } \\
\text { in the non- } \\
\text { responders } \\
\text { subgroup }\end{array}$ \\
\hline $\begin{array}{l}\text { Goran } \\
\quad \text { et al. }\end{array}$ & $\begin{array}{l}5 \text { women } \\
\mathrm{Age}=63(5) \\
\mathrm{BMl}=24(\mathrm{NR}) \\
6 \mathrm{men} \\
\mathrm{Age}=68(7) \\
\mathrm{BMl}=24(\mathrm{NR})\end{array}$ & $\begin{array}{l}\text { Cycle ergometer, } 3 \mathrm{~d} / \text { week. Progression from a } \\
\text { target EE of } 628 \mathrm{~kJ} / \mathrm{session} \text { at } 60 \% \mathrm{VO}_{2} \max \text { to } \\
1255 \mathrm{~kJ} / \mathrm{session} \text {, at } 85 \% \mathrm{VO}_{2} \max \end{array}$ & 8 Weeks & $\begin{array}{l}\text { DLW over } 10 \mathrm{~d} \text { at baseline and in } \\
\text { the last } 10 \mathrm{~d} \text { of the training } \\
\text { programme } \\
\text { REE by indirect calorimetry } \\
\text { ExEE by HR monitor } \\
\text { Non-ex PAEE }=\text { TEE }- \\
\text { (REE }+0 \cdot 1 \mathrm{TEE}+\text { ExEE) }\end{array}$ & $\begin{array}{l}\text { Non-exercise EE declined throughout the } \\
\text { intervention } \\
\text { No significant change in body mass }\end{array}$ & $\begin{array}{l}\text { Compensation: } \\
\text { decrease in NEAT }\end{array}$ \\
\hline $\begin{array}{l}\text { Herrmann } \\
\quad \text { et al. }\end{array}$ & $\begin{array}{l}74 \text { healthy, overweight/ } \\
\text { obese, sedentary men } \\
\text { and women } \\
\text { Responders } \\
\text { Age }=23 \text { (3) } \\
\mathrm{BMI}=31 \text { (4) }\end{array}$ & $\begin{array}{l}\text { Intervention: } \\
\text { Treadmill walking or jogging, } 5 \mathrm{~d} / \text { week, } 1674 \text { or } \\
2510 \mathrm{~kJ} / \text { session } \\
\text { Comparison between participants with weight } \\
\text { loss }<5 \% \text { (non-responders) } v . \geq 5 \% \\
\text { (responders) }\end{array}$ & 10 Months & $\begin{array}{l}\text { REE was assessed by indirect } \\
\text { calorimetry. TEE was assessed } \\
\text { using DLW over a } 14-d \text { period } \\
\text { (at baseline and at } 10 \text { months) } \\
\text { Non-Ex PAEE = (0.9 TEE-REE)- } \\
\text { net ExEE (i.e. ExEE-REE) }\end{array}$ & $\begin{array}{l}\text { Non-exercise EE and NEPA increased in } \\
\text { responders and decreased in non-responders } \\
\text { Sedentary time decreased in both groups, but } \\
\text { only in men was significant }\end{array}$ & $\begin{array}{l}\text { Compensation: NEAT } \\
\text { and NEPA } \\
\text { decreased in the } \\
\text { non-responders } \\
\text { group }\end{array}$ \\
\hline
\end{tabular}


Table 3. Continued

\begin{tabular}{|c|c|c|c|c|c|c|}
\hline Studies & Sample & Intervention & Length + follow-up & Measures & Results & Behavioural response \\
\hline & $\begin{array}{l}\text { Non-responders } \\
\mathrm{Age}=22(5) \\
\mathrm{BMI}=31(5)\end{array}$ & & & $\begin{array}{l}\text { NEPA and sedentary time were } \\
\text { assessed by an accelerometer } \\
\text { (model GT1M; Actigraph) }\end{array}$ & & \\
\hline $\begin{array}{l}\text { Hunter } \\
\quad \text { et al. }\end{array}$ & $\begin{array}{l}7 \text { women; } 8 \text { men } \\
\mathrm{Age}=67(4) \\
\mathrm{BMI}=25(4)\end{array}$ & $\begin{array}{l}\text { Supervised resistance training (RT), } 3 \text { times per } \\
\text { week for approximately } 45 \mathrm{~min} / \mathrm{session} \\
\text { Subjects were instructed to complete two sets of } \\
10 \text { repetitions in all exercises at an intensity of } \\
65-80 \% \text { of } 1 \text { RM with a } 2 \text {-min rest between } \\
\text { each set }\end{array}$ & 26 Weeks & $\begin{array}{l}\text { TEE assessed by DLW at baseline } \\
\text { and last } 2 \text { weeks of RT. } \\
\text { PAEE }=\text { TEE - REE - 0.1TEE } \\
\text { Adjusted PAEE = PAEE - } \\
\text { Average ExEE } \\
\text { Average ExEE assessed by } \\
\text { indirect calorimetry in a previous } \\
\text { study } \\
\text { ARTE index was used to derive } \\
\text { free-living PA (min/d) from PAEE }\end{array}$ & $\begin{array}{l}\text { TEE and TEE minus the average exercise EE } \\
\text { significantly increased } \\
\text { Free-living PA (via ARTE index) increased and } \\
\text { PAEE showed a tendency to raise } \\
\text { No significant weight change }\end{array}$ & $\begin{array}{l}\text { No compensation: no } \\
\text { changes in NEAT } \\
\text { and NEPA }\end{array}$ \\
\hline $\begin{array}{l}\text { Keytel } \\
\quad \text { et al. }\end{array}$ & $\begin{array}{l}\text { Control: } 10 \text { women } \\
\text { Age }=55(5) \\
\text { BMl }=27(4) \\
\text { Exercise: } 9 \text { women } \\
\text { Age }=58(7) \\
\text { BMI }=25(3)\end{array}$ & $\begin{array}{l}\text { Control: no exercise } \\
\text { Exercise: Walking, } 3 \mathrm{~d} / \text { week, } 70-75 \% \mathrm{HR}_{\max } \\
\text { beginning with } 3 \mathrm{~km} \text { walking on a sport's field } \\
\text { and progressing to walking or jogging } 3-6 \mathrm{~km} \\
\text { on the road }\end{array}$ & 8 Weeks & $\begin{array}{l}\text { Total daily energy expenditure } \\
\text { (TEE) was calculated from HR } \\
\text { minus EE regression equations } \\
\text { and } 24-\mathrm{h} \text { HR monitoring, at } \\
\text { baseline and end of intervention }\end{array}$ & $\begin{array}{l}\text { No significant differences in TEE either between } \\
\text { exercise and control, or before or after } \\
\text { exercise training } \\
\text { No significant differences in TEE on exercise } v \text {. } \\
\text { non-exercise days } \\
\text { Change in body weight not reported }\end{array}$ & $\begin{array}{l}\text { No compensation: no } \\
\text { change in NEAT }\end{array}$ \\
\hline $\begin{array}{l}\text { Leibel } \\
\quad \text { et al. }\end{array}$ & $\begin{array}{l}18 \text { obese }(11 \text { women and } \\
7 \text { men); } \\
\text { Age }=29(10) \\
\text { BMI }=(\mathrm{NR}) \\
23 \text { non-obese }(7 \text { women } \\
\text { and } 16 \text { men) } \\
\text { Age }=26(10) \\
\text { BMI }=(\mathrm{NR})\end{array}$ & $\begin{array}{l}\text { Diet intervention: liquid formula ( } 3347 \mathrm{~kJ} / \mathrm{d} 40 \% \\
\text { fat (maize oil), } 45 \% \text { carbohydrate (glucose } \\
\text { polymer), and } 15 \% \text { protein (casein } \\
\text { hydrolysate)). The energy intake was adjusted } \\
\text { until the body weight was constant for at least } \\
14 \mathrm{~d} \\
3 \text { groups lost weight (non-obese } 10 \% \text { loss, } n 11 \text {; } \\
\text { obese } 10 \% \text { loss, } n \text { 9; obese } 20 \% \text { loss, } n 12 \text { ) }\end{array}$ & $\begin{array}{l}\text { Weight loss } \\
\text { phase ranged } \\
\text { from } \\
4 \text { to } 7 \text { weeks } \\
\text { for the non- } \\
\text { obese and } \\
\text { from } 6 \text { to } \\
14 \text { weeks for } \\
\text { the obese }\end{array}$ & $\begin{array}{l}\text { TEE determined by DLW } \\
\text { REE and TEF measured by } \\
\text { indirect calorimetry in a } \\
\text { respiration chamber } \\
\text { Non-resting EE = TEE - } \\
\text { (REE }+ \text { TEF) } \\
\text { PA monitor by using a wall- } \\
\text { mounted radar detector in the } \\
\text { metabolic chamber }\end{array}$ & $\begin{array}{l}\text { Non-resting EE was significantly lower at } \\
\text { weights } 10 \text { and } 20 \% \text { below the initial weight } \\
\text { than at the initial weight } \\
\text { The percentage of time spent in motion during a } \\
\text { 23-h period did not differ }(n 8)\end{array}$ & $\begin{array}{l}\text { Compensation: } \\
\text { reduction in NEAT } \\
\text { No compensation: } \\
\text { no change in NEPA }\end{array}$ \\
\hline $\begin{array}{l}\text { Matsuo } \\
\text { et al. }\end{array}$ & $\begin{array}{l}90 \text { women } \\
\mathrm{Age}=52(7) \\
\mathrm{BMI}=28(3)\end{array}$ & $\begin{array}{l}\text { Combined diet and exercise intervention: } \\
\text { Diet: twelve } 90 \text {-min lectures + individual } \\
\text { counselling } \\
\text { Exercise: three } 60 \text {-min lectures. For a } \\
\text { sub-sample, additional supervised aerobic } \\
\text { exercise sessions ( } 90 \mathrm{~min} / \text { session, } \\
24 \text { sessions) }\end{array}$ & 14 Weeks & $\begin{array}{l}\text { Uniaxial accelerometer worn for } \\
7 \mathrm{~d} \text { at baseline and end of } \\
\text { intervention } \\
\text { PAEE did not discriminate between } \\
\text { training and spontaneous PA, } \\
\text { but one group did not have } \\
\text { supervised exercise sessions }\end{array}$ & $\begin{array}{l}\text { Significant increases were observed in daily } \\
\text { TEE/body weight and PAEE/body weight } \\
\text { A significant correlation was observed between } \\
\text { PAEE before and after the intervention } \\
\text { Significant decreases in body weight }\end{array}$ & $\begin{array}{l}\text { No compensation: no } \\
\text { change in NEAT }\end{array}$ \\
\hline $\begin{array}{l}\text { McLaughlin } \\
\text { et al. }^{(45)}\end{array}$ & $\begin{array}{l}\text { Eight men } \\
\text { Age }=23(1) \\
\mathrm{BMI}=25(5) \\
\text { Eight women } \\
\text { Age }=24(3) \\
\mathrm{BMI}=22(2)\end{array}$ & $\begin{array}{l}\text { Control phase: } 8 \mathrm{~d} \text { of usual activity } \\
\text { Exercise phase: } 8 \mathrm{~d} \text { with imposed cycle } \\
\text { ergometer to expend } 2092 \mathrm{~kJ} \text { plus REE on } 4 \\
\text { alternate days of the week }\end{array}$ & $16 d$ & $\begin{array}{l}\text { HR monitoring with individuals HR/ } \\
\text { EE calibration }\end{array}$ & $\begin{array}{l}\text { No significant differences in non-exercise PAEE } \\
\text { between exercise and control periods } \\
\text { Body mass did not change }\end{array}$ & $\begin{array}{l}\text { No compensation: no } \\
\text { change in NEAT }\end{array}$ \\
\hline $\begin{array}{l}\text { Meijer } \\
\quad \text { et al. }\end{array}$ & $\begin{array}{l}16 \text { women } \\
\mathrm{Age}=35(4) \\
\mathrm{BMl}=23(2) \\
16 \text { men } \\
\mathrm{Age}=37(3) \\
\mathrm{BMI}=23(2)\end{array}$ & $\begin{array}{l}\text { Running programme: } 1 \text { supervised }+ \\
3 \text { non-supervised sessions, for a total distance } \\
\text { of } 15-25 \mathrm{~km} / \text { week after } 8 \text { weeks and } \\
25-40 \mathrm{~km} / \text { week after } 20 \text { weeks } \\
\text { Training included long slow-distance running } \\
\text { (at } 70 \%-80 \% \mathrm{HR}_{\max } \text { ), running at a higher }\end{array}$ & 5 Months & $\begin{array}{l}\text { Accelerometer for } 7 \text { consecutive } \\
\text { days at baseline and } 20 \text { weeks } \\
\text { DLW for TEE assessment } \\
\text { SMR by indirect calorimetry } \\
\text { Non-ExEE assessed from non- } \\
\text { exercise activities }\end{array}$ & $\begin{array}{l}\text { Accelerometer counts excluding training-related } \\
\text { increased approximately } 15 \% \text { in men, but this } \\
\text { change was not significant } \\
\text { In men, a discrepancy was observed between } \\
\text { the increase of TEE and the expenditure due } \\
\text { to ExEE and non-ExEE activities. When }\end{array}$ & $\begin{array}{l}\text { No compensation: no } \\
\text { change in NEAT } \\
\text { and NEPA }\end{array}$ \\
\hline
\end{tabular}




\begin{tabular}{|c|c|c|c|c|c|c|}
\hline Studies & Sample & Intervention & Length + follow-up & Measures & Results & Behavioural response \\
\hline & & $\begin{array}{l}\text { speed }\left(80-95 \% \mathrm{HR}_{\max }\right) \text {, and interval training } \\
\left(95-100 \% \mathrm{HR}_{\max }\right)\end{array}$ & & & $\begin{array}{l}\text { expressing TEE normalised for body mass or } \\
\text { FFM, this difference disappeared } \\
\text { Change in body mass not reported }\end{array}$ & \\
\hline $\begin{array}{l}\text { Meijer } \\
\quad \text { et al. }\end{array}$ & $\begin{array}{l}\text { Control: } 4 \text { women; } 3 \text { men. } \\
\text { Age }=57(3) \\
\text { BMl }=25(1) \\
\text { Exercise: } 8 \text { women; } \\
7 \text { men. } \\
\text { Age }=59(4) \\
\mathrm{BMl}=26(3)\end{array}$ & $\begin{array}{l}\text { Supervised exercise, twice a week: a group } \\
\text { session of } 60-\text { min with various aerobic } \\
\text { exercises on } 1 \mathrm{~d} \text { and an individual session of } \\
90 \text { min consisting of } 9 \text { exercises using cardio- } \\
\text { and weight-stack machines on day 2; at } 50 \% \\
\text { HR reserve }\end{array}$ & 12 Weeks & $\begin{array}{l}\text { Tri-axial accelerometer worn for } \\
14 \mathrm{~d} \text { at baseline, } 6 \text { and } 12 \text { weeks }\end{array}$ & $\begin{array}{l}\text { At week } 6, \text { no significant differences in non- } \\
\text { exercise PA between training days and non- } \\
\text { training days } \\
\text { At week } 12 \text {, after adjusting for training activity, } \\
\text { physical activity on training days was } \\
\text { significantly lower than on non-training days } \\
\text { No change in body mass in both groups }\end{array}$ & $\begin{array}{l}\text { Compensation: } \\
\text { reduction in NEPA }\end{array}$ \\
\hline $\begin{array}{l}\text { Meijer } \\
\quad \text { et al. }\end{array}$ & $\begin{array}{l}\text { Control: } 5 \text { women; } 6 \text { men. } \\
\text { Age }=59(4) \\
\text { BMl }=26(3) \\
\text { Exercise: } 11 \text { women; } \\
11 \text { men. } \\
\text { Age }=63(8) \\
\mathrm{BMI}=29(4)\end{array}$ & $\begin{array}{l}\text { Supervised exercise, twice a week: a group } \\
\text { session of } 60 \text { min with various aerobic } \\
\text { exercises on } 1 \mathrm{~d} \text { and an individual session of } \\
90 \text {-min consisting of } 9 \text { exercises using cardio- } \\
\text { and weight-stack machines on day } 2 \text {. Intensity } \\
50 \% \text { HR reserve }\end{array}$ & 12 Weeks & $\begin{array}{l}\text { Tri-axial accelerometer worn for } \\
14 \mathrm{~d} \text { at baseline, } 6 \text { and } 12 \text { weeks }\end{array}$ & $\begin{array}{l}\text { At week } 6 \text {, no significant differences in non- } \\
\text { exercise accelerometer data between training } \\
\text { days and non-training days } \\
\text { At week } 12 \text {, after adjusting for training activity, } \\
\text { physical activity on training days was } \\
\text { significantly lower than on non-training days } \\
\text { No change in body mass in both groups }\end{array}$ & $\begin{array}{l}\text { Compensation: } \\
\text { reduction in NEPA }\end{array}$ \\
\hline $\begin{array}{l}\text { Van Dale } \\
\quad \text { et al. }{ }^{(49)}\end{array}$ & $\begin{array}{l}\text { Diet: } 6 \text { women } \\
\text { Age }=20-45 \\
\text { BMI }=30(1) \\
\text { Diet + Exercise: } 6 \text { women } \\
\text { Age }=20-45 \\
\mathrm{BMI}=30(1)\end{array}$ & $\begin{array}{l}\text { Diet (D): low energy formula diet first } 4 \text { weeks } \\
\text { (approximately } 2929 \mathrm{~kJ} / \mathrm{d} \text { ); combination of the } \\
\text { diet with normal food on the next } 8 \text { weeks } \\
\text { (approximately } 3347 \mathrm{~kJ} / \mathrm{d} \text { ) } \\
\text { Diet + Exercise (DE): low-energy formula diet first } \\
4 \text { weeks (approximately } 2929 \mathrm{~kJ} / \mathrm{d} \text { ); } \\
\text { combination of the diet with normal food on the } \\
\text { next } 8 \text { weeks }(3602 \mathrm{~kJ} / \mathrm{d} \text { ) plus } 4 \text { exercise } \\
\text { sessions, } 2-\mathrm{h} \text { aerobic training and } 2-\mathrm{h} \text { fitness } \\
\text { training per week at } 55 \% \mathrm{VO}_{2 \max }\end{array}$ & 12 Weeks & $\begin{array}{l}\text { HR memory system measured 24- } \\
\text { h EE at baseline, weeks } 4 \text { and } \\
\text { Wrist accelerometer Actometer } \\
\text { worn over } 5 \text { full consecutive } \\
\text { days, including } 2 \mathrm{~d} \text { of training } \\
\text { and weekend days }\end{array}$ & $\begin{array}{l}\text { PA increased } 27 \% \text { in the DE group but not in the } \\
\text { D group } \\
\text { The adoption of regular structured exercise did } \\
\text { not result in a negative compensatory } \\
\text { reduction in NEPA } \\
\text { No significant weight loss in both groups }\end{array}$ & $\begin{array}{l}\text { No compensation: } \\
\text { increases in NEPA }\end{array}$ \\
\hline $\begin{array}{l}\text { Van Etten } \\
\quad \text { et al. }{ }^{(50)}\end{array}$ & $\begin{aligned} & \text { Control: } 8 \text { men } \\
& \text { Age }=35(6) \\
& \text { BMI }=24(\mathrm{NR}) \\
& \text { Exercise: } 18 \text { men } \\
& \text { Age }=33(6) \\
& \text { BMl }=24 \text { (NR) }\end{aligned}$ & $\begin{array}{l}\text { Control: no exercise } \\
\text { Exercise: supervised RT, } 2 \text { non-consecutive } \\
\quad \text { days/week, } 3 \text { sets, } 15 \text { reps, } 10 \text { exercises }\end{array}$ & 18 weeks & $\begin{array}{l}\text { Tri-axial accelerometer worn for } \\
7 \mathrm{~d} \text {, at baseline and } \\
\text { intervention's end } \\
\text { DLW over a 2-week period on } 12 \\
\text { exercisers } \\
\text { Indirect calorimetry for SMR } \\
\text { HR monitor used to determine } \\
\text { ExEE } \\
\text { Non-ex PAEE }=\text { TEE- } \\
\text { SMR - } 0.1 \mathrm{TEE} \text { - ExEE }\end{array}$ & $\begin{array}{l}\text { Non-training PA measured by accelerometer did } \\
\text { not change between groups } \\
\text { Non-training EE measured did not change } \\
\text { between groups } \\
\text { Body mass did not change in both groups }\end{array}$ & $\begin{array}{l}\text { No compensation: no } \\
\text { change in NEAT } \\
\text { and NEPA }\end{array}$ \\
\hline $\begin{array}{l}\text { Weinsier } \\
\text { et al. }{ }^{(51)}\end{array}$ & $\begin{array}{l}18 \text { white and } 14 \text { black } \\
\text { women } \\
\text { Age }=38(7) \\
\mathrm{BMI}=29(2)\end{array}$ & $\begin{array}{l}\text { Energy-restricted diet without exercise: diet } \\
\text { provided a fixed proportion of carbohydrate, } \\
\text { fat and protein ( } 55,22 \text { and } 23 \% \text {, respectively) } \\
\text { and had an energy content of } 3347 \mathrm{~kJ} / \mathrm{d} \\
\text { No attempt was made to alter the subjects' self- } \\
\text { selected patterns of PA }\end{array}$ & $\begin{array}{l}\text { Length } \\
\text { necessary to } \\
\text { achieve the } \\
\text { weight loss of } \\
10 \mathrm{~kg} \text { (average } \\
24 \text { weeks) }\end{array}$ & $\begin{array}{l}\text { REE measured in a chamber } \\
\text { calorimeter } \\
\text { TEE measured by DLW over } 14 \mathrm{~d} \\
\text { PAEE was assessed as } \\
\text { TEE - 0.1TEE - REE } \\
\text { Free-living PA was derived using } \\
\text { ARTE index }\end{array}$ & $\begin{array}{l}\text { Free-living PA was similar between the groups. } \\
\text { Free-living PAEE did not change significantly } \\
\text { after weight loss } \\
\text { Free-living PA increased an average of } 30 \% \\
\text { above-rest, but not above-sleep. However, } \\
\text { reported activities did not change from before } \\
\text { to after weight loss }\end{array}$ & $\begin{array}{l}\text { No compensation: no } \\
\text { change in NEAT or } \\
\text { NEPA }\end{array}$ \\
\hline
\end{tabular}

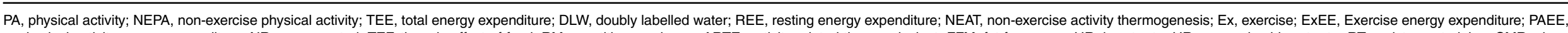

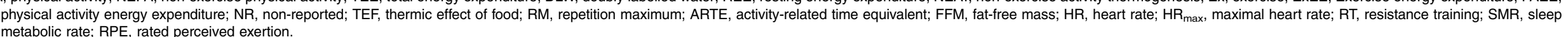


Table 4. Diet-only, exercise-only, combined diet and exercise and all studies combined, according to the presence or absence of changes in non-exercise activity thermogenesis (NEAT) and non-exercise physical activity (NEPA)

(Medians and ranges; numbers and percentages)

\begin{tabular}{|c|c|c|c|c|c|c|c|c|}
\hline \multirow[b]{3}{*}{ All studies $(n 36)^{*}$} & \multicolumn{4}{|c|}{ Reduction ( $n$ 15) } & \multicolumn{4}{|c|}{ No changes ( $n$ 21) } \\
\hline & \multicolumn{2}{|c|}{ NEAT $(n 11)$} & \multicolumn{2}{|c|}{ NEPA $(n 7)$} & \multicolumn{2}{|c|}{ NEAT $(n$ 13) } & \multicolumn{2}{|c|}{ NEPA $(n 17)$} \\
\hline & Median & Range & Median & Range & Median & Range & Median & Range \\
\hline Age (years) & 39 & $23-66$ & 39 & $23-63$ & 43 & $22-71$ & 41 & $22-71$ \\
\hline BMI $\left(\mathrm{kg} / \mathrm{m}^{2}\right)$ & 30 & $23-37$ & 29 & $25-37$ & 30 & $23-44$ & 30 & $23-44$ \\
\hline Study length (months) & 5 & $2-12$ & 4 & $2-10$ & 5 & $0 \cdot 3-10$ & 6 & $0 \cdot 3-12$ \\
\hline Weight loss $(\mathrm{kg})$ & -10 & $-29-4$ & -8 & $-27-0$ & -5 & $-14-0$ & -7 & $-29-0$ \\
\hline Behavioural therapy (studies) & \multirow{2}{*}{\multicolumn{2}{|c|}{3}} & \multirow{3}{*}{\multicolumn{2}{|c|}{1}} & \multirow{3}{*}{\multicolumn{2}{|c|}{3}} & \multirow{3}{*}{\multicolumn{2}{|c|}{6}} \\
\hline n & & & & & & & & \\
\hline Diet-only studies & & & & & & & & \\
\hline Age (years) & 39 & $25-59$ & 44 & $39-53$ & 38 & $33-51$ & 38 & $25-59$ \\
\hline $\mathrm{BMI}\left(\mathrm{kg} / \mathrm{m}^{2}\right)$ & 28 & $27-37$ & 35 & $28-37$ & 31 & $28-44$ & 28 & $27-44$ \\
\hline Study length (months) & 6 & $3-12$ & 4 & $2-4$ & 5 & $0.3-10$ & 6 & $0 \cdot 3-12$ \\
\hline Weight loss (\%) & -10 & $-29-3.5$ & -17 & $-27-8 \cdot 3$ & -8 & $-14-0$ & -8 & $-29-0$ \\
\hline Behavioural therapy (studies) & & & & & & & & \\
\hline n & \multicolumn{2}{|c|}{3} & \multirow{2}{*}{\multicolumn{2}{|c|}{1}} & \multirow{2}{*}{\multicolumn{2}{|c|}{1}} & \multirow{2}{*}{\multicolumn{2}{|c|}{3}} \\
\hline Exercise-only studies & & & & & & & & \\
\hline Age (years) & 49 & $23-66$ & 40 & $23-63$ & 48 & $22-67$ & 50 & $22-67$ \\
\hline $\mathrm{BMI}\left(\mathrm{kg} / \mathrm{m}^{2}\right)$ & 29 & $23-34$ & 26 & $25-31$ & 29 & $23-35$ & 30 & $23-35$ \\
\hline Study length (months) & 3 & $2-10$ & 6 & $3-10$ & 6 & $0 \cdot 3-10$ & 6 & $0.3-10$ \\
\hline Weight loss (\%) & -8 & $-8-7$ & -1 & $-8-0$ & -1 & $-9-0$ & -1 & $-9-1$ \\
\hline Behavioural therapy (studies) & \multirow{2}{*}{\multicolumn{2}{|c|}{0}} & \multirow{2}{*}{\multicolumn{2}{|c|}{0}} & \multirow{2}{*}{\multicolumn{2}{|c|}{0}} & \multirow{2}{*}{\multicolumn{2}{|c|}{1}} \\
\hline n & & & & & & & & \\
\hline Exercise duration (min/session) & 53 & $45-60$ & 75 & $60-90$ & 45 & $20-120$ & 45 & $20-120$ \\
\hline Exercise frequency (times/week) & 3 & $2-10$ & 6 & $3-10$ & 6 & $0.3-10$ & 6 & $0.3-10$ \\
\hline Aerobic exercise (\%) & \multicolumn{2}{|c|}{100} & \multicolumn{2}{|c|}{71} & \multicolumn{2}{|c|}{76} & \multicolumn{2}{|c|}{87} \\
\hline Strength exercise $(\%)$ & \multicolumn{2}{|c|}{0} & & & \multicolumn{2}{|c|}{18} & & \\
\hline Combined aerobic and strength (\%) & & & & & & & & \\
\hline Combined diet and exercise studies & & & & & & & & \\
\hline Age (years) & 59 & $39-59$ & & & 43 & $33-71$ & 48 & 33-71 \\
\hline $\mathrm{BMI}\left(\mathrm{kg} / \mathrm{m}^{2}\right)$ & 33 & $32-33$ & & & 32 & $28-44$ & 33 & $28-44$ \\
\hline Study length (months) & 5 & $2-5$ & & & 5 & $3-6$ & 5 & 2-6 \\
\hline Weight loss (\%) & -12 & $-15-9$ & & & -9 & $-13-5$ & -9 & $-15-5$ \\
\hline Behavioural therapy (studies) & & & & & & & & \\
\hline n & & & & & & & & \\
\hline Exercise duration (min/session) & 30 & $30-55$ & & & 50 & $30-90$ & 48 & $30-90$ \\
\hline Exercise frequency (times/week) & 3 & $2-3$ & & & 4 & $2-5$ & 4 & $2-5$ \\
\hline Aerobic exercise $(\%)$ & & & & & & & & \\
\hline Strength exercise $(\%)$ & & & & & & & & \\
\hline Combined aerobic and strength (\%) & & & & & & & & \\
\hline
\end{tabular}

* Discrepancy between the number of overall studies that showed reductions (15) or no changes (21) in NEAT and NEPA and the number of studies displayed in the row below, according-the presence or absence of changes in NEAT (11 v. 13, respectively) and NEPA ( 7 v. 17, correspondingly), is due to studies that determined both NEAT and NEPA.

prescribed) by subtracting the sum of REE by indirect calorimetry with TEF (assumed as 0.1TEE) from TEE obtained by DLW.

Methods for assessing non-exercise physical activity. In RCT, Martin et $a l .{ }^{(16)}$ assessed NEPA with ACC (model 716 (Actigraph) and RT3 accelerometer (Stayhealthy, Inc.)). In another trial under the CALERIE study, Martin et al. ${ }^{(22)}$ used a metabolic chamber to assess NEPA by determining the percent time participants were active. In RT, DeLany et al. ${ }^{(28)}$ assessed NEPA by counting steps/d using multisensor PA monitors (SenseWearPro3; BodyMedia Inc.). Racette et al. ${ }^{(14)}$ assessed NEPA from HR monitors for PA assessment (excluding exercise data on exercise activity). Weigle ${ }^{(34)}$ used a pedometer for assessing NEPA. In NRT, Bonomi et al. ${ }^{(35)}$ assessed NEPA through a combined actometer and Doppler measures. Brehm et al. ${ }^{(27)}$ used pedometers for assessing NEPA.

De Groot et $a l .{ }^{(37)}$ assessed NEPA using an actometer and Doppler metre counts. Leibel et al. ${ }^{(43)}$ assessed NEPA using a respiratory chamber equipped with a wall-mounted radar detector to monitor PA. Van Dale et al. ${ }^{(49)}$ assessed NEPA using an actometer and HR monitor. Weinsier et al. ${ }^{(51)}$ determined NEPA by using the activity-related time equivalent (Arte) index for free-living PA ( $\mathrm{min} / \mathrm{d})$.

Weight loss. Median weight loss was $-11.0 \mathrm{~kg}$ (range -29.2 to $0 \cdot 1$ ) with a median of $-12 \cdot 0 \mathrm{~kg}$ (range $-29 \cdot 2$ to $0 \cdot 1$ ) for NRT, $-13 \cdot 0$ (range -26.9 to $-6 \cdot 1$ ) for RT and -8.4 (range $-11 \cdot 2$ to -3.5 ) for RCT.

Risk of bias. The quality of assessment tool rated one trial as weak $^{(43)}$, twelve as moderate ${ }^{(14-16,22,27,29,33-35,37,49,51)}$ and one trial as strong ${ }^{(28)}$ (online Supplementary material SIII).

Main outcome. A total of fifteen out of twenty-four intervention arms (seven out of fourteen diet-only interventions) reported a significant decrease in NEAT or NEPA resulting from the prescribed diet. 
Among the nine diet-only trials assessing NEAT, decreases were observed in six studies (fourteen diet-only trial arms) specifically three intervention arms of an $\mathrm{NRT}^{(43)}$, four intervention arms of $\mathrm{RT}^{(14,33,34)}$ and seven intervention arms of $\operatorname{RCT}^{(15,16)}$

From the eleven studies assessing NEPA, behavioural compensation was observed in three diet-only interventions (four intervention arms), specifically one intervention arm of an $\mathrm{NRT}^{(37)}$ and three intervention arms of $\mathrm{RT}^{(14,34)}$.

\section{Exercise-only interventions}

The thirty-five exercise-only intervention arms (twenty studies) comprised approximately $56 \%$ of the total number of studies included in this review, with a total of eleven NRT (55\%), two RT (10\%) and seven RCT (35\%).

\section{Study characteristics}

Sample size. Exercise-only studies comprised 917 participants with a median sample size of 28 (range 8-139). NRT included a median sample size of 20 (range 9-40), RT included a median sample size of 27 (range 26-28) and RCT included a median sample size of 35 (range 8-139).

Completion rate. Fourteen trials reported the percentage of exercise sessions attended as $>99^{(19,21)}, 62 \cdot 5^{(26,40)}, 94^{(24)}, 99 \%$ for moderate intensity and $96 \%$ for high intensity ${ }^{(23)}, 90 \%$ for aerobic training, $84 \%$ for combined aerobic and resistance training, but \% was not available for resistance training ${ }^{(31)}$, $100 \%$ for $30-\mathrm{min}$ and $60-80 \%$ for $60-$ and $90-$ min groups $^{(32)}$, $86^{(38,48)},>90^{(41)}, 85^{(47)}, 95^{(50)}, 100^{(45)}$ and $64 \%(26,40)$, whereas one trial $^{(36)}$ reported the level of exercise EE (prescribed $6276 \mathrm{~kJ} /$ week; achieved $6000 \mathrm{~kJ} /$ week). Whybrow et al. ${ }^{(25)}$ and Meijer et $a l .{ }^{(46)}$ reported good compliance, but no percent values were given. Compliance with the exercise protocol was not reported in three trials ${ }^{(20,39,42)}$.

Trial length. The median duration of the studies was $5 \cdot 3$ (range $0 \cdot 3-10$ ) months, varying from $4 \cdot 2$ (range $0 \cdot 3-10$ ) for NRT, 8 months for the two RT and $5 \cdot 2$ (range 0.5-10) for the RCT.

Behavioural intervention. Only the study of Kozey-Keadle et $a{ }^{(21)}$ included behavioural therapy.

Exercise mode. NRT included a variety of indoor or outdoor walking/running ${ }^{(36,38,42,46)}$, cycle ergometer exercise $^{(39,45)}$, fitness classes and resistance training ${ }^{(47,48)}$, resistance training only $^{(41,50)}$ and treadmill ${ }^{(40)}$. RT intervention arms involved a combination of resistance and aerobic training ${ }^{(31)}$ and daily walking $^{(32)}$. The remaining RCT intervention arms used laboratorybased aerobic exercise conducted on cycle ergometers/rowers/ steppers/Arc Trainer/treadmills ${ }^{(19-21,23,25,26)}$, or outdoor walking/ running $^{(24)}$.

Exercise supervision. All exercise sessions were supervised in thirteen NRT trials ${ }^{(39-41,47,48,50)}$ and $\mathrm{RCT}^{(19-21,23-26)}$, partially supervised in five NRT trials ${ }^{(36,38,42,45,46)}$ or not reported in $\mathrm{RT}^{(31,32)}$.

Exercise prescription (frequency). The median exercise frequency was $4 \cdot 1$ (range $2 \cdot 0-7 \cdot 0$ ) d/week with daily week values of 3.4 (range $2 \cdot 0-5 \cdot 0$ ) for NRT, 3.9 (range $2.5-5 \cdot 0$ ) for RT and $4 \cdot 8$ (range $3 \cdot 5-7 \cdot 0$ ) for RCT.
Exercise prescription (intensity). Six NRT-prescribed intensity as a percentage of maximal $\mathrm{VO}_{2 \max }\left(53 \%{ }^{(45)}, 85 \%{ }^{(39)}\right)$, percentage of maximal HR (70-80\% $\left.\mathrm{HR}_{\max }^{(40)}, 70-75 \%{ }^{(42)}\right)$, by level of EE relative to body weight (moderate intensity: $28.6 \mathrm{~kJ} / \mathrm{kg}$ and high intensity: $57 \cdot 1 \mathrm{~kJ} / \mathrm{kg})^{(25)}$, one based on HR reserve $(50 \%$ $\mathrm{HRR}^{(48)}$ ) and one based on ratings of perceived exertion (11-13 on a 15 -point scale $\left.{ }^{(38)}\right)$. Resistance training in the study by Hunter et $a l .{ }^{(41)}$ was conducted at $65-85 \%$ of one maximum repetition. Exercise intensity in RT/RCT studies that included aerobic exercise was prescribed as a percentage of maximal/ peak $\mathrm{VO}_{2}$ in five trials $\left(50 \%{ }^{(19)}\right.$; three sessions $>70 \%$ and remaining self-selected ${ }^{(23)}, 70 \%{ }^{(24)} ; 65-80 \%$ - vigorous and $40-55$ - moderate $\left.{ }^{(20)}, 75 \%^{(31)}\right)$, maximum HR $\left(70-80 \%{ }^{(26)}\right)$, between 40 and $65 \% \mathrm{HR}$ reserve ${ }^{(21)}$. Prescribed exercise intensity was not reported in four trials ${ }^{(36,46,47,50)}$ and selfselected in an $\mathrm{RT}^{(32)}$. Resistance training was performed at 8-12 repetition maximum (approximately $80 \%)^{(31)}$, whereas Van Etten et al. ${ }^{(50)}$ did not report intensity.

Exercise prescription (duration). Median session duration was approximately $55 \mathrm{~min}(20-120)$, with values of about $50 \mathrm{~min}(35-75)$ in NRT, $67 \mathrm{~min}(30-111)$ and $52 \mathrm{~min}(20-120)$. Four NRT-prescribed exercise duration was performed by time $\left(50 \mathrm{~min}^{(38)}\right.$, $60 \mathrm{~min}$ aerobic plus $90 \mathrm{~min}$ aerobic/strength ${ }^{(47)}$, $60 \mathrm{~min}$ aerobic plus $90 \mathrm{~min}$ aerobic/strength ${ }^{(48)}$ and 39 and $55 \mathrm{~min}^{(40)}$, respectively, for the $1674 \mathrm{~kJ} /$ session and $2510 \mathrm{~kJ} / \mathrm{ses}-$ sion), three based on level of exercise EE (1569-2092 kJ/ses$\operatorname{sion}^{(36)}, 1255 \mathrm{~kJ} /$ session $^{(39)}, 2092 \mathrm{~kJ} /$ session $^{(45)}$ ) and two based on walking/running distance ${ }^{(42,46)}$. The median duration of exercise for the five trials prescribing exercise by time was 150 (range 60200) $\mathrm{min} /$ week. The median for trials prescribing exercise by EE was 4094 (range 1883-6276) kJ/week. Prescribed walking distance was $3-6 \mathrm{~km} / \mathrm{d}^{(42)}$, and running distance was $25-40 \mathrm{~km} /$ week $^{(46)}$. One study dosed exercise by level of EE relative to body weight 28.6 or $\left.57.1 \mathrm{~kJ} / \mathrm{kg}^{(25)}\right)$ or absolute EE $(2092 \mathrm{~kJ} / \mathrm{d}$ above $\left.\mathrm{REE}^{(45)}\right)$. In three trials, prescribed exercise duration was calculated as EE per body weight ${ }^{(19,20,31)}$, four based on time $e^{(21,24,25,32)}$ and two according to level of exercise EE (1255 and $2510 \mathrm{~kJ} /$ session $^{(23)} ; 1674$ and $2510 \mathrm{~kJ} /$ session $^{(26)}$ ). In three RCT, exercise time was prescribed for $60^{(24)}, 40^{(21)}$ and $40 \mathrm{~min}^{(25)}$. Schutz et al. ${ }^{(32)}$ involved three arms with different durations (30, 60 and $90 \mathrm{~min}$ ). Willis et al. ${ }^{(26)}$ prescribed 39 and $55 \mathrm{~min}$ for the $1674 \mathrm{~kJ} /$ session and $2510 \mathrm{~kJ} / \mathrm{session}$; Rosenkilde et al. $^{(23)}$ prescribed 1255 and $2510 \mathrm{~kJ} / \mathrm{d}$; Church et al. ${ }^{(19)}$ prescribed 17,33 and $50 \mathrm{~kJ} / \mathrm{kg}$ per week; and Rangan et al. ${ }^{(31)}$ and Hollowell et al. ${ }^{(20)}$ dosed duration as $58.6 \mathrm{~kJ} / \mathrm{kg}$ per week and 59 and $96 \mathrm{~kJ} / \mathrm{kg}$ per week. Resistance training in the study of Van Etten et al. ${ }^{(50)}$ consisted of three sets of fifteen reps over ten exercises, whereas for Hunter et al. ${ }^{(41)}$ duration of $45 \mathrm{~min}$, ten repetitions with $2 \mathrm{~min}$ of rest by set was used.

\section{Participant characteristics}

Age. Studies were generally conducted in adults with a median age of $44 \cdot 1$ years $(22 \cdot 1-66 \cdot 8)$, specifically $49 \cdot 0$ years (range 22.1-66.8) for NRT, $38 \cdot 7$ years (range $27 \cdot 0-52$ ) for RT and 42.2 years (range $23-57.5$ ) for RCT.

Sex. Five studies included women only ${ }^{(19,32,36,38,42)}$, three studies included men only ${ }^{(23,24,50)}$ and twelve studies included a combined sample of women and men ${ }^{(20,21,25,26,31,39-41,45-48)}$. 
$B M I$. Nine studies included overweight/obese individuals ${ }^{(19-21,24,26,31,38,40,48)}$. Non-obese participants were included in ten studies ${ }^{(23,25,32,39,41,42,45-47,50)}$. Obese-only individuals were included in one study ${ }^{(36)}$. Median BMI was $31.2 \mathrm{~kg} / \mathrm{m}^{2}$ (range 27.4-43.6) in the studies that provided data on this parameter, with a median of $27.0 \mathrm{~kg} / \mathrm{m}^{2}$ (range $22.8-33.9$ ) for NRT, $27.8 \mathrm{~kg} / \mathrm{m}^{2}$ (range 22.8-33.9) for RT and $42.2 \mathrm{~kg} / \mathrm{m}^{2}$ (range 23.0-57.9) for RCT.

Ethnicity. Two studies described the ethnicity representation in the study sample as Caucasian ${ }^{(23,41)}$, whereas three studies included Caucasian, Black, Hispanics and Asians ${ }^{(19,26,40)}$. The remaining studies did not report ethnicity.

Physical activity level/fitness. In all, seventeen studies characterised the level of PA of the participants as sedentary or exercising $<2-3 \mathrm{~d} /$ week $^{(19-21,23-26,31,36,40-42,45-48,50)}$. Di Blasio et $a l .{ }^{(38)}$ reported that PA level ranged from sedentary to highly active and fitness level from poor to good. Two studies did not report PA level or fitness ${ }^{(32,39)}$

Methods for assessing non-exercise activity thermogenesis. Hollowell et $a l^{(20)}$ used an ACC (RT3 accelerometer; Stayhealthy, Inc.) for assessing NEAT (although authors referred 'non-exercise PAEE') by excluding exercise EE (ExEE) (including the $30 \mathrm{~min}$ before and after exercise). Turner et al. ${ }^{(24)}$ assessed NEAT (referred to as 'non-prescribed PAEE') through a combined HR monitor and ACC (Actiheart; CamNtech Ltd) by, respectively, subtracting the ExEE from the overall PAEE. Whybrow et al. ${ }^{(25)}$ calculated non-exercise EE as the difference between TEE from DLW and ExEE by an individual calibrated HR monitor. Willis et al. ${ }^{(26)}$ determined NEAT (referred as 'nonexercise EE') as (0.9 TEE-RMR)-net ExEE (ExEE-RMR), where TEE was assessed by DLW and REE and ExEE by indirect calorimetry. Rangan et al. ${ }^{(31)}$ assessed NEAT (referred to as nonexercise PAEE) with ACC (RT3 accelerometer; Stayhealthy, Inc.) by removing exercise data EE. Across NRT, Colley et al. ${ }^{(36)}$ assessed NEAT by subtracting the sum of exercise EE (using HR monitor), REE (indirect calorimetry) and TEF (assumed as 10\% of TEE) from TEE obtained by DLW. Di Blasio et al. ${ }^{(38)}$ used a SenseWear Pro 2 armband on training and non-training days to assess whether PAEE between responders and non-responders differed in non-exercise days. Goran \& Poehlman ${ }^{(39)}$ used DLW (TEE), indirect calorimetry (REE) and HR monitoring during exercise training to determine NEAT as TEE-(REE + 0.1TEE + ExEE). Herrman et al. ${ }^{(40)}$ assessed NEAT (referred to as "nonexercise EE') as (0.9 TEE-RMR)-net Exercise EE (exercise EERMR), where TEE was assessed by DLW and REE and ExEE by indirect calorimetry. Hunter et $a .^{(41)}$ used DLW (TEE) and indirect calorimetry (REE) to determine PAEE (as TEE-01TEEREE) and adjusted PAEE (adjusted for energy cost of average ExEE). HR monitoring with individual HR/EE calibration was used by Keytel et al. ${ }^{(42)}$ to assess 24 -h daily EE between training and non-training days and between exercise and control group. McLaughlin et al. ${ }^{(45)}$ calculated NEAT from HR monitor using HR/EE individual calibration between control and exercise periods. Meijer et al. ${ }^{(46)}$ assessed TEE with DLW, SMR by indirect calorimetry and NEAT (referred as EE from nonexercise activities). Van Etten et al. ${ }^{(50)}$ assessed NEAT (referred to as non-training EE) as TEE from DLW, minus SMR from indirect calorimetry minus ExEE from HR measurements.
Methods for assessing non-exercise physical activity. Church et $a{ }^{(19)}$ used pedometers outside the training sessions for assessing NEPA. Kozey-Keadle et al. ${ }^{(21)}$ assessed NEPA through ActivPAL (PAL Technologies). In RT, Schutz et al. ${ }^{(32)}$ determined NEPA through steps/d using an ACC (uniaxial), calculating the ratio between expected (from baseline steps/d) and observed steps/d from the prescribed walking. Rosenkilde et al. ${ }^{(23)}$ assessed NEPA through ACC (model GT1M; Actigraph), by subtracting exercise counts from PA counts. Turner et al. ${ }^{(24)}$ assessed NEPA (as the time spent participating in PA above predetermined thresholds) by a combined HR monitor and ACC (Actiheart; CamNtech Ltd) after subtracting exercise activity. Willis et al. ${ }^{(26)}$ determined NEPA was assessed through ACC (model GT1M; Actigraph) by subtracting ACC data from the exercise training sessions. In the studies of Meijer et al. ${ }^{(46-48)}$, NEPA was assessed by ACC. Herrman et al. ${ }^{(40)}$ determined NEPA from ACC (model GT1M; Actigraph) after removing ACC data from the exercise sessions. Hunter et al. ${ }^{(41)}$ used an arte index for free-living PA $(\mathrm{min} / \mathrm{d})$ that reflects the amount of time a person spend in free-living PA. Van Etten et al. ${ }^{(50)}$ assessed NEPA through a triaxial ACC.

Weight loss. Median weight loss was $-2.3 \mathrm{~kg}$ (range -9.1 to $0 \cdot 1$ ) with a median and range of $-2 \cdot 1 \mathrm{~kg}$ (range $-9 \cdot 1$ to $0 \cdot 1$ ) for NRT and $-2.4 \mathrm{~kg}$ (range -5.2 to -0.8 ) for RCT (data not available for RT).

Risk of bias. Using the quality assessment tool, eight trials were rated as weak $^{(20,24,25,31,32,36,42,45)}$, eleven as moderate $^{(21,23,26,38-41,46-48,50)}$ and one trial as strong ${ }^{(19)}$ (online Supplementary material SIII).

Main outcome. A total of seven out of twenty studies (eight in thirty-five intervention arms) reported a significant decrease in NEPA or NEAT, resulting from the prescribed exercise.

From the fourteen exercise-only interventions, reductions in NEAT were observed in four NRT (four intervention arms) ${ }^{(36,38-40)}$.

In a total of twelve exercise-only trials, decreases in NEPA were observed in four studies, specifically three arms of NRT ${ }^{(40,47,48)}$ and two arms of a $\mathrm{RT}^{(32)}$.

\section{Combined diet and exercise interventions}

The 11 combined diet and exercise interventions arms ( 9 studies) comprised $25 \%$ of the total number of studies included in this review with a total of 2 NRT (22\%), 5 RT (56\%) and 2 RCT (22\%).

\section{Study characteristics}

Sample size. Combined diet and exercise trials comprised a total of 244 participants with a median sample size of 23.8 (range 5-90). NRT included a median sample of 48 (range 6-90), RT of 20 (range 7-61) and two RCT, from the same large study (CALERIE), included twelve participants.

Completion rate. Two studies reported compliance to the exercise sessions $\left(>90^{(33)}\right.$ and $\left.22 \%{ }^{(28)}\right)$. Two studies reported compliance to diet $\left(100^{(33)}\right.$ and $\left.55 \%{ }^{(28)}\right)$. The remaining studies did not include data on compliance to either exercise or diet.

Trial length. The median duration of the studies was 4.4 months (range 2-0-6.0). Median duration was $2 \cdot 8$ months 
(range 2.5-3.0) for NRT, 4.4 months (range 2.0-6.0) for RT and 6 months for the two RCT.

Behavioural intervention. A total of five studies included behavioural therapy ${ }^{(14,15,22,30,44)}$ comprising $44 \%$ of the combined diet and exercise studies included in this review.

Energy restriction. EI was reduced by $12 \cdot 5 \%{ }^{(15,22)}$ and $75 \%$ of $\mathrm{RMR}^{(14)}$. EI was prescribed as $5021 \mathrm{~kJ} / \mathrm{d}^{(44)}$, approximately $2092 \mathrm{~kJ} / \mathrm{d}$ in the first 4 weeks followed by 4 weeks at approximately $3515 \mathrm{~kJ} / \mathrm{d}^{(29)}$, about $2929 \mathrm{~kJ} / \mathrm{d}$ in the first 4 weeks and about $3602 \mathrm{~kJ} / \mathrm{d}$ in the next 8 weeks ${ }^{(49)}$, and according to body weight $(<90.7 \mathrm{~kg}, \quad 5021-6276 \mathrm{~kJ} / \mathrm{d} ; \quad>90.7 \mathrm{~kg}$ and $<113.4 \mathrm{~kg}, 6276-7531 \mathrm{~kJ} / \mathrm{d}$; and $>113.4 \mathrm{~kg}, 7531-8368 \mathrm{~kJ} / \mathrm{d}^{(28)}$ ). EI was also prescribed as a reduction of $2510 \mathrm{~kJ} / \mathrm{d}^{(30)}$ and $1443 \mathrm{~kJ} / \mathrm{d}^{(33)}$

Exercise mode. One study used indoor aerobic and strength training ${ }^{(29)}$, indoor aerobic/fitness training ${ }^{(44,49)}$, treadmill/ walking $^{(30,33)}$, treadmill/stairstep/rowing/bicycling ${ }^{(14)}$, walking/ running/bicycling ${ }^{(15,22)}$ and outdoor brisk walking ${ }^{(28)}$.

Exercise supervision. Exercise sessions were supervised in $\mathrm{NRT}^{(44,49)}$ and $\mathrm{RT}^{(14,30)}$, partially supervised in $\mathrm{RT}^{(29)}$ and $\mathrm{RCT}^{(15,22)}$, or not reported in two $\mathrm{RT}^{(28,33)}$.

Exercise prescription (frequency). The median exercise frequency was $3.7 \mathrm{~d} /$ week (range $2 \cdot 0-5 \cdot 0$ ) with $3.0 \mathrm{~d} /$ week (range $2 \cdot 0-4 \cdot 0$ ) for NRT, $3 \cdot 6 \mathrm{~d} /$ week (range $3 \cdot 0-5 \cdot 0$ ) for RT and $5 \mathrm{~d} /$ week in the two RCT.

Exercise prescription (intensity). Three trials prescribed intensity as a percentage of maximal $\mathrm{VO}_{2 \max }$ (NRT: $55 \%{ }^{(49)}$; RT: $\left.50-60 \%{ }^{(29)} ; 60-65 \%^{(14)}\right)$, and two based on HR reserve $\left(65-70 \%{ }^{(30)} ; 45-50 \%\right.$; and $\left.70-75 \%{ }^{(33)}\right)$. Prescribed exercise intensity was not reported in one trial ${ }^{(44)}$ and was self-selected in three trials ${ }^{(15,22,28)}$

Exercise prescription (duration). Exercise duration was prescribed by time in most studies (NRT: $90 \mathrm{~min} / \mathrm{session}^{(44)}$ and $60 \mathrm{~min} / \mathrm{session}^{(49)}$; RT: $30 \mathrm{~min} / \operatorname{session}^{(30)}, 55$ and $30 \mathrm{~min} / \operatorname{session}^{(33)}$; $60 \mathrm{~min} / \mathrm{session}^{(28)}$; $45 \mathrm{~min} / \operatorname{session}^{(14)}$; $90 \mathrm{~min} /$ session $\left.{ }^{(29)}\right)$. Prescribed exercise duration was self-selected in two $\operatorname{RCT}^{(15,22)}$.

\section{Participant characteristics}

Age. Studies were generally conducted in adults with a median age of $44 \cdot 1$ years (range $22 \cdot 1-66 \cdot 8$ ), specifically $49 \cdot 0$ years (range $22 \cdot 1-66 \cdot 8$ ) for NRT, $38 \cdot 7$ years (range $27 \cdot 0-52$ ) for RT and 42.2 years (range $23-57.5$ ) for the two RCT.

Sex. Five studies included women only ${ }^{(14,29,33,44,49)}$, and four studies included a combined sample of women and men ${ }^{(15,22,28,30)}$.

BMI. Three studies included overweight/obese individuals $^{(33,44,49)}$, four studies included obese participants ${ }^{(14,28-30)}$ and two trials included non-obese individuals ${ }^{(15,22)}$. In the studies that provided data on this parameter, BMI was $32.3 \mathrm{~kg} / \mathrm{m}^{2}$ (range $27.5-43.6$ ), with a median and range of $29 \cdot 1 \mathrm{~kg} / \mathrm{m}^{2}$ (range $27 \cdot 8-30 \cdot 3$ ) for NRT, $34.6 \mathrm{~kg} / \mathrm{m}^{2}$ (range 32.4 43.6) for RT and $27.7 \mathrm{~kg} / \mathrm{m}^{2}$ (range $27.5-27 \cdot 8$ ) for the two RCT.

Ethnicity. Two studies include all ethnic groups - that is, Caucasian, Black, Asian and Hispanics ${ }^{(15,22)}$; one study included Asians only ${ }^{(44)}$; three studies included Caucasian and Black participants ${ }^{(28,30,33)}$; and three studies did not report ethnic groups $^{(14,29,49)}$
Participant activity level/fitness. Three studies characterised the level of PA of the participants as sedentary ${ }^{(14,30,33)}$.

Methods for assessing non-exercise activity thermogenesis. Redman et $a l .{ }^{(15)}$ assessed NEAT (referred as activity-related EE) as the residual value of the regression between measured TEE obtained from DLW and measured SMR using indirect calorimetry, but ExEE was not assessed. DeLany et al. ${ }^{(28)}$ assessed NEAT (referred to as PAEE) as TEE from DLW minus the sum of REE by indirect calorimetry with TEF (assumed as 0.1TEE) but ExEE was not calculated, restricting an accurate assessment of NEAT. Kempen et al. ${ }^{(29)}$ assessed NEAT (referred to as non-exercised PA) by subtracting the sum of SMR from indirect calorimetry plus TEF (assumed as 0.1TEE) plus ExEE (from HR monitor) from TEE by DLW. Racette et $a l .{ }^{(14)}$ assessed NEAT (referred to as non-exercise PAEE) with DLW (for TEE), TEF and REE by indirect calorimetry and ExEE from HR monitors as TEE - (REE + TEF + ExEE). Wang et al. ${ }^{(33)}$ used ACC (RT3 accelerometer; Stayhealthy, Inc.) for non-exercise PAEE (excluding exercise data on EE). Matsuo et al. ${ }^{(44)}$ assessed NEAT using an ACC (Lifecorder; Suzuken Co. Ltd).

Methods for assessing non-exercise physical activity. Martin et $a{ }^{(22)}$ used a metabolic chamber to assess NEPA by determining the percent time participants were active. DeLany et al. ${ }^{(28)}$ assessed NEPA through steps/d using a multisensor PA monitor. Nicklas et al. ${ }^{(30)}$ used an ACC for assessing NEPA. Racette et $a l .{ }^{(14)}$ assessed NEPA from HR monitors for PA assessment (excluding exercise data on exercise activity). Van Dale et al. ${ }^{(49)}$ assessed NEPA using an actometer and HR monitor.

Weight loss. Median weight loss was $-9.8 \mathrm{~kg}$ (range -14.8 to $-5 \cdot 2$ ) - specifically $-9 \cdot 2 \mathrm{~kg}$ (range -13.2 to $-5 \cdot 2$ ) for NRT, $-10.4 \mathrm{~kg}$ (range -14.8 to -6.6 ) for $\mathrm{RT}$ and $-8.8 \mathrm{~kg}$ for the two RCT.

Risk of bias. On the basis of the quality assessment tool, one trial was rated as weak ${ }^{(44)}$, seven as moderate $(14,15,22,29,30,33,49)$ and one trial as strong ${ }^{(28)}$ (online Supplementary material SIII).

Main outcome. A total of two out of nine combined diet and exercise interventions (three in eleven intervention arms) reported a significant decrease in NEPA or NEAT resulting from the prescribed diet plus exercise.

From the six combined diet and exercise trials that assessed NEAT, reductions were observed in three intervention arms of two $\mathrm{RT}^{(29,33)}$, whereas no behavioural compensation was observed in the five interventions that assessed NEPA.

\section{Discussion}

We systematically reviewed thirty-six studies with a variety of designs including NRT and RT to address whether the prescribed diet and/or exercise led to reductions in NEPA/NEAT in healthy adults. A reduction in NEAT has been hypothesised as a way to compensate for the increased EE of prescribed exercise and/or energy deficit from energetic restriction diets, resulting in less-than-expected negative energy balance and related weight loss ${ }^{(17)}$.

Overall, our review found decreases in NEPA or NEAT in fifteen out of thirty-six studies conducted in healthy adults using 
diet-only intervention, combined diet and exercise intervention and exercise-only intervention (twenty-six out of a total of seventy intervention arms). Decreases in NEPA and/or NEAT were observed in seven out of fourteen diet-only interventions, two out of nine combined diet and exercise trials and seven out twenty exercise-only trials. In addition, it is important to highlight some other relevant findings. This review reported that the intervention arms that decreased NEAT were the ones presenting higher median values of weight loss (approximately $10 \mathrm{~kg}$ ) compared with those who reported no changes in NEAT (approximately $5 \mathrm{~kg}$ ). This observation suggests that reductions in NEAT may play a protective role when substantial body weight is lost.

Only seven of twenty exercise-only studies (eight out thirtyfive intervention arms $-23 \%$ ) included in this review reported a significant decrease in NEAT assessed by DLW/HR/metabolic chambers/cart ${ }^{(36,38-40)}$ or NEPA assessed by pedometer/ ACC/actometer/doppler ${ }^{(32,40,47,48)}$. Studies that reported decreased NEPA/NEAT used a non-randomised design and were mainly conducted in sedentary overweight or obese adults. Age varied from young ${ }^{(32,40)}$, middle-aged ${ }^{(36,38,47,48)}$ to older adults ${ }^{(39)}$. We observed that median age was similar between those who compensated compared with those who did not compensate, although Washburn et al. ${ }^{(10)}$ suggest that NEPA/NEAT may decrease in response to exercise training in older individuals.

In exercise-only studies that showed reductions in NEAT, the median duration of the studies was half the median duration of trials that did not present behavioural compensation. Apparently, compensation seems to occur in exercise studies of reduced duration. These results do not extend the findings observed by Riou et $a l .{ }^{(53)}$, reporting that the degree of energy compensation is near $84 \%$ for exercise interventions of a longer duration.

In contrast, seven out fourteen studies (fifteen out twentyfour intervention arms - 63\%) testing the effects of diet-only interventions reported a significant decrease in NEAT assessed by DLW/HR/ ACC/metabolic chambers/cart ${ }^{(14-16,33,34,43)}$ or NEPA assessed by pedometer/ACC/actometer/doppler ${ }^{(14,34,37)}$. Studies that reported decreased NEPA/NEAT were conducted in sedentary overweight or obese adults and used a randomised design. Median age was below 40 years $^{(14-16,37,43)}$ in the majority of the trials, but middle-aged to older adults were studied $^{(33,34)}$

Considering the combined effects of diet and exercise, only two out of nine studies (three out eleven intervention arms $27 \%$ ) testing the effects of diet plus exercise interventions showed a reduction in NEAT ${ }^{(29,33)}$ but not in NEPA by means of DLW/ACC/metabolic carts ${ }^{(29,33)}$. Studies that reported reductions in NEAT were conducted in sedentary overweight or obese adults and used a randomised design with a median age of 37 years $^{(29)}$ and approximately 60 years ${ }^{(33)}$.

Reductions in NEAT/NEPA were observed in more than half of the diet-only intervention arms (approximately 63\%), followed by diet plus exercise (27\%) and exercise-only (23\%) intervention arms. It is possible that diet-only interventions are more prone to cause reductions in NEAT/NEPA compared with exercise-only or diet plus exercise, but this hypothesis has not been evaluated in a trial comparing changes in NEPA/NEAT in response to diet, exercise training protocols and combined diet and exercise training protocols. Moreover, in studies that involved exercise-only and diet plus exercise studies, the decrease in NEAT was absent in trials that prescribed resistance training. These observations suggest that exercise prescription may indeed have benefits for weight management interventions, although well-designed trials are required to definitively clarify the role of exercise dose on NEAT and NEPA.

Further, considering all the intervention arms that presented behavioural compensation in free-living PA, approximately $81 \%$ reduced NEAT and only $19 \%$ presented decrements in NEPA (twenty-one and nine intervention arms, respectively, out of twenty-six). Indeed, studies using methods to assess both NEAT and NEPA found reductions in the former but not in the latter $^{(16)}$. These observations may be owing to methodological limitations in assessing NEPA in free-living conditions, although we only included those studies that used objective measures of PA. Indeed, obtaining accurate measures of NEPA and NEAT in free-living conditions is challenging, specifically during an energy balance intervention given the variable nature of human adaptive response. DLW method and activity monitors are the most common approaches ${ }^{(20,38,39,54)}$. DLW is the state-of-the-art method for measuring TEE ${ }^{(54)}$. When DLW is used in exercise training trials, NEAT is typically estimated using the measured or estimated REE and ExEE. Most of the studies assume that the TEF represents $10 \%$ of TEE without changes over the intervention. Therefore, NEAT is calculated as the difference between the TEE and the sum of REE, TEF (or the assumption of $0 \cdot 1$ TEE) and ExEE prescribed in exercise or combined diet and exercise interventions. In diet-only studies, authors refer to NEAT as PAEE, assuming that participants did not engage in exercise activities outside the energy-restricted intervention. A major drawback of determining NEAT is the involved cost, specifically owing to the use of DLW measurements, limiting the number of participants included in the studies. Nevertheless, DLW provides one value of TEE over a period of days, which means that for assessing NEAT when exercise is prescribed the related EE needs to be accounted for. In addition, DLW does not provide the type of non-Ex PA performed (i.e. sitting and ambulatory movement) or PA patterns. These limitations can partly be overcome using activity monitors, but estimates of EE from accelerometry are less accurate than those from $\mathrm{DLW}^{(54)}$. Indeed, PAEE might be somewhat independent of measurements of body movement for wide ranges of PA amounts ${ }^{(55)}$. Pontzer et al. ${ }^{(55)}$ observed that after controlling for body composition and size TEE was positively related with $\mathrm{PA}$, but the association was stronger over the lower range of PA, whereas TEE plateaued in individuals whose PA was considered in the upper range, supporting a constrained TEE model.

\section{Limitations of the studies}

There are important short-comings in the studies included in this systematic review. The methodological issues in assessing PA and EE, described previously, may limit the accuracy in 
evaluating the impact of diet and/or exercise training on NEAT/ NEPA. Considering the relevance of energy balance interventions for weight management, it is important to assess the effect of diet and/or exercise training on compensatory responses using accurate techniques. Only fourteen out of thirty-six studies $^{(14-16,22,25,28,29,36,39-41,46,50,51)}$ assessed NEAT using DLW, the state-of-the-art method for TEE measurements in freeliving individuals ${ }^{(56)}$. In addition, for determining NEAT, REE measures through indirect calorimetry and accurate methods for assessing ExEE are required. Only two studies included in this review provided measures of exercise EE by indirect calorimetry $^{(26,40)}$, although REE was assessed with this technique in eleven studies along with $\mathrm{DLW}^{(14,15,22,28,29,36,39,41,46,50,51)}$.

Studies were not specifically designed and appropriately powered to detect differences in NEAT/NEPA between- or within-group with statistical significance in response to diet and/or exercise. The majority of these trials were conducted in small samples of $<20$ participants ${ }^{(14,24,29,34,36,37,39,41,42,45,47,49,50)}$. In addition, in those studies that were specifically designed to address the effect of diet and/or exercise training on NEAT/ NEPA, small samples were used ${ }^{(15,22,24,50)}$

Other limitations include the lack of studies that tested the impact of the degree of energy restriction, weight-loss magnitude and exercise dose.

\section{Limitations of this review}

The findings of our review are based on data coming essentially from weak to moderate study designs (NRT and RT with an elevated risk of bias).

\section{Conclusions}

Although the present systematic review did not find evidence to suggest that diet and/or exercise training has a significant effect, decreases in NEPA (four studies), NEAT (eight studies) or both (three studies) were observed in $63 \%$ of the total diet-only intervention arms, with only 23 and $27 \%$ of the declines observed in exercise-only or combined diet and exercise trial arms. We also reported that participants who decreased NEAT presented a median amount of weight loss that was almost double the amount of those participants who did not compensate, suggesting that behavioural compensation leading to reductions in NEAT may depend on the degree of energy stores used when substantial body weight is lost, thus conserving energy.

Nevertheless, additional RT designed to specifically evaluate the impact of diet and/or exercise on NEPA/NEAT should be conducted in overweight/obese adults. In particular, studies should be powered to detect clinically significant differences. In addition, measures of daily and exercise EE should be included for an accurate assessment of NEAT. Studies must also analyse the impact of the degree of energy restriction, weight-loss magnitude, exercise dose and participant characteristics in more detail.

\section{Acknowledgements}

The data sets generated for this analysis are available from the corresponding author on reasonable request.
The current work was supported by National funding from the Portuguese Foundation for Science and Technology within the R\&D units 472 (UID/DTP/00447/2013). P. B. J. is supported by the Portuguese Foundation for Science and Technology with a post-doctoral scholarship (SFRH/BPD/115977/2016).

A. M. S., P. J. T., N. K. and L. B. S. designed the study. A. M. S. performed the literature searches. P. B. J. and E. V. C. conducted the title, abstract and full text screening and data. All authors contributed to the interpretation of the results and critically reviewed the manuscript. All authors read and approved the final manuscript.

The authors declare that there are no conflicts of interest.

\section{Supplementary material}

For supplementary material/s referred to in this article, please visit https://doi.org/10.1017/S000711451800096X

\section{References}

1. Hall KD, Heymsfield SB, Kemnitz JW, et al. (2012) Energy balance and its components: implications for body weight regulation. Am J Clin Nutr 95, 989-994.

2. King NA, Caudwell P, Hopkins M, et al. (2007) Metabolic and behavioral compensatory responses to exercise interventions: barriers to weight loss. Obesity (Silver Spring) 15, $1373-1383$

3. Dulloo AG, Jacquet J, Montani JP, et al. (2012) Adaptive thermogenesis in human body weight regulation: more of a concept than a measurable entity? Obes Rev 13, Suppl. 2, 105-121.

4. Knuth ND, Johannsen DL, Tamboli RA, et al. (2014) Metabolic adaptation following massive weight loss is related to the degree of energy imbalance and changes in circulating leptin. Obesity (Silver Spring) 22, 2563-2569.

5. Hall KD, Sacks G, Chandramohan D, et al. (2011) Quantification of the effect of energy imbalance on bodyweight. Lancet 378, 826-837.

6. Thomas DM, Martin CK, Heymsfield S, et al. (2011) A simple model predicting individual weight change in humans. $J$ Biol Dyn 5, 579-599.

7. Melanson EL, Keadle SK, Donnelly JE, et al. (2013) Resistance to exercise-induced weight loss: compensatory behavioral adaptations. Med Sci Sports Exerc 45, 1600-1609.

8. Levine JA (2004) Nonexercise activity thermogenesis (NEAT): environment and biology. Am J Physiol Endocrinol Metab 286, E675-E685.

9. Fedewa MV, Hathaway ED, Williams TD, et al. (2017) Effect of exercise training on non-exercise physical activity: a systematic review and meta-analysis of randomized controlled trials. Sports Med 47, 1171-1182.

10. Washburn RA, Lambourne K, Szabo AN, et al. (2014) Does increased prescribed exercise alter non-exercise physical activity/energy expenditure in healthy adults? A systematic review. Clin Obes 4, 1-20.

11. Levine JA, Lanningham-Foster LM, McCrady SK, et al. (2005) Interindividual variation in posture allocation: possible role in human obesity. Science 307, 584-586.

12. Heyman MB, Young VR, Fuss P, et al. (1992) Underfeeding and body weight regulation in normal-weight young men. $\mathrm{Am}$ J Physiol 263, R250-R257.

13. Velthuis-te Wierik EJ, Westerterp KR \& van den Berg H (1995) Impact of a moderately energy-restricted diet on energy 
metabolism and body composition in non-obese men. Int J Obes Relat Metab Disord 19, 318-324.

14. Racette SB, Schoeller DA, Kushner RF, et al. (1995) Effects of aerobic exercise and dietary carbohydrate on energy expenditure and body composition during weight reduction in obese women. Am J Clin Nutr 61, 486-494.

15. Redman LM, Heilbronn LK, Martin CK, et al. (2009) Metabolic and behavioral compensations in response to caloric restriction: implications for the maintenance of weight loss. PLOS ONE 4, e4377.

16. Martin CK, Das SK, Lindblad L, et al. (2011) Effect of calorie restriction on the free-living physical activity levels of nonobese humans: results of three randomized trials. $J$ Appl Physiol (1985) 110, 956-963.

17. Dhurandhar EJ, Kaiser KA, Dawson JA, et al. (2015) Predicting adult weight change in the real world: a systematic review and meta-analysis accounting for compensatory changes in energy intake or expenditure. Int J Obes (Lond) 39, 1181-1187.

18. Liberati A, Altman DG, Tetzlaff J, et al. (2009) The PRISMA statement for reporting systematic reviews and meta-analyses of studies that evaluate health care interventions: explanation and elaboration. PLoS Med 6, e1000100.

19. Church TS, Martin CK, Thompson AM, et al. (2009) Changes in weight, waist circumference and compensatory responses with different doses of exercise among sedentary, overweight postmenopausal women. PLOS ONE 4, e4515.

20. Hollowell RP, Willis LH, Slentz CA, et al. (2009) Effects of exercise training amount on physical activity energy expenditure. Med Sci Sports Exerc 41, 1640-1644.

21. Kozey-Keadle S, Staudenmayer J, Libertine A, et al. (2014) Changes in sedentary time and physical activity in response to an exercise training and/or lifestyle intervention. J Phys Act Health 11, 1324-1333.

22. Martin CK, Heilbronn LK, de Jonge L, et al. (2007) Effect of calorie restriction on resting metabolic rate and spontaneous physical activity. Obesity (Silver Spring) 15, 2964-2973.

23. Rosenkilde M, Auerbach P, Reichkendler MH, et al. (2012) Body fat loss and compensatory mechanisms in response to different doses of aerobic exercise - a randomized controlled trial in overweight sedentary males. Am J Physiol Regul Integr Comp Physiol 303, R571-R579.

24. Turner JE, Markovitch D, Betts JA, et al. (2010) Nonprescribed physical activity energy expenditure is maintained with structured exercise and implicates a compensatory increase in energy intake. Am J Clin Nutr 92, 1009-1016.

25. Whybrow S, Hughes DA, Ritz P, et al. (2008) The effect of an incremental increase in exercise on appetite, eating behaviour and energy balance in lean men and women feeding ad libitum. Br J Nutr 100, 1109-1115.

26. Willis EA, Herrmann SD, Honas JJ, et al. (2014) Nonexercise energy expenditure and physical activity in the Midwest Exercise Trial 2. Med Sci Sports Exerc 46, 2286-2294.

27. Brehm BJ, Spang SE, Lattin BL, et al. (2005) The role of energy expenditure in the differential weight loss in obese women on low-fat and low-carbohydrate diets. J Clin Endocrinol Metab 90, $1475-1482$.

28. DeLany JP, Kelley DE, Hames KC, et al. (2014) Effect of physical activity on weight loss, energy expenditure, and energy intake during diet induced weight loss. Obesity (Silver Spring) 22, 363-370.

29. Kempen KP, Saris WH \& Westerterp KR (1995) Energy balance during an 8-wk energy-restricted diet with and without exercise in obese women. Am J Clin Nutr 62, 722-729.

30. Nicklas BJ, Gaukstern JE, Beavers KM, et al. (2014) Selfmonitoring of spontaneous physical activity and sedentary behavior to prevent weight regain in older adults. Obesity (Silver Spring) 22, 1406-1412.

31. Rangan VV, Willis LH, Slentz CA, et al. (2011) Effects of an 8-month exercise training program on off-exercise physical activity. Med Sci Sports Exerc 43, 1744-1751.

32. Schutz Y, Nguyen DM, Byrne NM, et al. (2014) Effectiveness of three different walking prescription durations on total physical activity in normal- and overweight women. Obes Facts 7, 264-273.

33. Wang X, Lyles MF, You T, et al. (2008) Weight regain is related to decreases in physical activity during weight loss. Med Sci Sports Exerc 40, 1781-1788.

34. Weigle DS (1988) Contribution of decreased body mass to diminished thermic effect of exercise in reduced-obese men. Int J Obes 12, 567-578.

35. Bonomi AG, Soenen S, Goris AHC, et al. (2013) Weight-loss induced changes in physical activity and activity energy expenditure in overweight and obese subjects before and after energy restriction. PLOS ONE 8, e59641.

36. Colley RC, Hills AP, King NA, et al. (2010) Exercise-induced energy expenditure: implications for exercise prescription and obesity. Patient Educ Couns 79, 327-332.

37. De Groot LCPGM, Van Es AJH, Van Raaij JMA, et al. (1990) Energy metabolism of overweight women 1 mo and 1 y after an 8-week slimming period. Am J Clin Nutr 51, 578-583.

38. Di Blasio A, Ripari P, Bucci I, et al. (2012) Walking training in postmenopause: effects on both spontaneous physical activity and training-induced body adaptations. Menopause 19, 23-32.

39. Goran MI \& Poehlman ET (1992) Endurance training does not enhance total energy expenditure in healthy elderly persons. Am J Physiol 263, E950-E957.

40. Herrmann SD, Willis EA, Honas JJ, et al. (2015) Energy intake, nonexercise physical activity, and weight loss in responders and nonresponders: The Midwest Exercise Trial 2. Obesity (Silver Spring) 23, 1539-1549.

41. Hunter GR, Byrne NM, Sirikul B, et al. (2008) Resistance training conserves fat-free mass and resting energy expenditure following weight loss. Obesity (Silver Spring) 16, 1045-1051.

42. Keytel LR, Lambert MI, Johnson J, et al. (2001) Free living energy expenditure in post menopausal women before and after exercise training. Int J Sport Nutr Exerc Metab 11, 226-237.

43. Leibel RL, Rosenbaum M \& Hirsch J (1995) Changes in energy expenditure resulting from altered body weight. $N$ Engl J Med 332, 621-628.

44. Matsuo T, Okura T, Nakata Y, et al. (2007) The influence of physical activity-induced energy expenditure on the variance in body weight change among individuals during a diet intervention. Obes Res Clin Pract 1, 109-117.

45. McLaughlin R, Malkova D \& Nimmo MA (2006) Spontaneous activity responses to exercise in males and females. Eur J Clin Nutr 60, 1055-1061.

46. Meijer GA, Janssen GM, Westerterp KR, et al. (1991) The effect of a 5-month endurance-training programme on physical activity: evidence for a sex-difference in the metabolic response to exercise. Eur J Appl Physiol Occup Physiol 62, $11-17$.

47. Meijer EP, Westerterp KR \& Verstappen FT (1999) Effect of exercise training on total daily physical activity in elderly humans. Eur J Appl Physiol Occup Physiol 80, 16-21.

48. Meijer EP, Westerterp KR \& Verstappen FT (2000) Effect of exercise training on physical activity and substrate utilization in the elderly. Int J Sports Med 21, 499-504.

49. Van Dale D, Schoffelen PFM, Ten Hoor F, et al. (1989) Effects of addition of exercise to energy restriction on 24-hour energy 
expenditure, sleeping metabolic rate and daily physical activity. Eur J Clin Nutr 43, 441-451.

50. Van Etten LM, Westerterp KR, Verstappen FT, et al. (1997) Effect of an 18-wk weight-training program on energy expenditure and physical activity. J Appl Physiol (1985) 82, 298-304.

51. Weinsier RL, Hunter GR, Zuckerman PA, et al. (2000) Energy expenditure and free-living physical activity in black and white women: Comparison before and after weight loss. Am J Clin Nutr 71, 1138-1146.

52. Armijo-Olivo S, Stiles CR, Hagen NA, et al. (2012) Assessment of study quality for systematic reviews: a comparison of the Cochrane Collaboration Risk of Bias Tool and the Effective Public Health Practice Project Quality
Assessment Tool: methodological research. I Eval Clin Pract 18, $12-18$

53. Riou ME, Jomphe-Tremblay S, Lamothe G, et al. (2015) Predictors of Energy compensation during exercise interventions: a systematic review. Nutrients 7, 3677-3704.

54. Westerterp KR (2009) Assessment of physical activity: a critical appraisal. Eur J Appl Physiol 105, 823-828.

55. Pontzer H, Durazo-Arvizu R, Dugas LR, et al. (2016) Constrained total energy expenditure and metabolic adaptation to physical activity in adult humans. Curr Biol 26, 410-417.

56. Schoeller DA (1999) Recent advances from application of doubly labeled water to measurement of human energy expenditure. J Nutr 129, 1765-1768. 